\title{
Inhibitory synchrony as a mechanism for attentional gain modulation is
}

\author{
Paul H. Tiesinga ${ }^{\text {a,* }}$, Jean-Marc Fellous ${ }^{\text {b,c, }, \text {, Emilio Salinas }}{ }^{\text {d }}$, Jorge V. José e , \\ Terrence J. Sejnowski ${ }^{\text {b,c,f,g }}$ \\ ${ }^{a}$ Department of Physics and Astronomy, University of North Carolina, Campus Box 3255, Chapel Hill, NC 27599-3255, United States \\ ${ }^{\mathrm{b}}$ Computational Neurobiology Lab, Salk Institute, La Jolla, CA 92037, United States \\ ${ }^{\mathrm{c}}$ Howard Hughes Medical Institute, Salk Institute, La Jolla, CA 92037, United States \\ ${ }^{\mathrm{d}}$ Department of Neurobiology and Anatomy, Wake Forest University School of Medicine, Winston-Salem, NC 27157, United States \\ ${ }^{\text {e } C e n t e r ~ f o r ~ t h e ~ I n t e r d i s c i p l i n a r y ~ R e s e a r c h ~ o n ~ C o m p l e x ~ S y s t e m s ~ a n d ~ P h y s i c s ~ D e p a r t m e n t, ~ N o r t h e a s t e r n ~ U n i v e r s i t y, ~ B o s t o n, ~ M A ~ 02115, ~ U n i t e d ~ S t a t e s ~}$ \\ ${ }^{\mathrm{f}}$ Sloan-Swartz Center for Theoretical Neurobiology, Salk Institute, La Jolla, CA 92037, United States \\ ${ }^{\mathrm{g}}$ Division of Biological Sciences, University of California at San Diego, La Jolla, CA 92093, United States
}

\begin{abstract}
Recordings from area V4 of monkeys have revealed that when the focus of attention is on a visual stimulus within the receptive field of a cortical neuron, two distinct changes can occur: The firing rate of the neuron can change and there can be an increase in the coherence between spikes and the local field potential (LFP) in the gamma-frequency range $(30-50 \mathrm{~Hz})$. The hypothesis explored here is that these observed effects of attention could be a consequence of changes in the synchrony of local interneuron networks. We performed computer simulations of a Hodgkin-Huxley type neuron driven by a constant depolarizing current, $I$, representing visual stimulation and a modulatory inhibitory input representing the effects of attention via local interneuron networks. We observed that the neuron's firing rate and the coherence of its output spike train with the synaptic inputs was modulated by the degree of synchrony of the inhibitory inputs. When inhibitory synchrony increased, the coherence of spiking model neurons with the synaptic input increased, but the firing rate either increased or remained the same. The mean number of synchronous inhibitory inputs was a key determinant of the shape of the firing rate versus current $(f-I)$ curves. For a large number of inhibitory inputs $(\sim 50)$, the $f-I$ curve saturated for large $I$ and an increase in input synchrony resulted in a shift of sensitivity - the model neuron responded to weaker inputs $I$. For a small number $(\sim 10)$, the $f-I$ curves were non-saturating and an increase in input synchrony led to an increase in the gain of the response-the firing rate in response to the same input was multiplied by an approximately constant factor. The firing rate modulation with inhibitory synchrony was highest when the input network oscillated in the gamma frequency range. Thus, the observed changes in firing rate and coherence of neurons in the visual cortex could be controlled by top-down inputs that regulated the coherence in the activity of a local inhibitory network discharging at gamma frequencies.
\end{abstract}

(C) 2005 Elsevier Ltd. All rights reserved.

Keywords: Selective attention; Synchrony; Noise; Gamma oscillation; Gain modulation; Computer model

\footnotetext{
Work supported in part by startup funds from the University of North Carolina and the Sloan-Swartz Center for Theoretical Neurobiology at the Salk Institute (PT); By startup funds from Wake Forest University and NINDS grant NS044894-01 (ES); By the CIRCS fund at Northeastern University (JVJ); Also by the Howard Hughes Medical Institute at the Salk Institute (JMF, TJS).

* Corresponding author. Tel.: +1 919962 7199; fax: +1 9199620480 E-mail address: tiesinga@physics.unc.edu (P.H. Tiesinga).

URL: http://www.neuro.physics.unc.edu/ (P.H. Tiesinga).

${ }^{1}$ Present address: JMF, Biomedical Engineering department, Duke University, Durham, NC 27708-0281, USA.
}

\section{Introduction}

Only a small part of the information in natural visual scenes is consciously accessible following a brief presentation $[61,69]$. The information that is retained depends on what is attended [34]. The neural correlates of selective attention have been studied in monkeys using recordings from single neurons $[16,17,29,44,48,49,53,63,64,82]$. A key finding is that attention modulates both the mean firing 
rate of a neuron in response to a stimulus $[49,63,64]$ and the coherence of spikes with other neurons responsive to the stimulus $[29,70]$. The increase of coherence with attention is strongest in the gamma frequency range [25].

There are many different types of inhibitory interneurons in the cortex, each with different patterns of input and output. For example, the basket cells project almost exclusively onto the somas and proximal dendrites of pyramidal neurons. In addition to their function in suppressing cortical activity, inhibitory cells may also be responsible for shaping the temporal pattern of spiking activity in the cortical network. In particular, the synapses from baskets cells near the spike initiating zone are effective in gating the occurrence of spikes $[15,45]$, and since a single basket cell has multiple inhibitory contacts on several thousand pyramidal cells, it could synchronize a subset of active cortical neurons.

Local cortical interneurons are not isolated from each other but form networks connected by fast GABAergic inhibitory synapses and electrical gap junctions $[6,31-33,35]$. Networks of inhibitory interneurons have been implicated in the generation of synchronous gamma-frequency-range oscillations in the hippocampus $[28,39,81,88]$ and the cortex [21], and could entrain a large number of principal cells $[12,33,43,71]$ as well as modulate their firing rates [3]. Hence, interneuron networks could mediate the effects of attention observed in cortical neurons.

We recently proposed a mechanism, synchrony by competition, for rapid synchrony modulation in interneuron networks [79] (see Section 4), that could explain how attention modulates the synchrony of interneuron networks. The focus of this study is on the impact of synchronous inhibitory input onto the principal output neurons of the cortex.

The degree of synchrony of inhibitory inputs can be characterized by their temporal dispersion, referred to here as jitter. A lower value of jitter corresponds to more synchrony and means that inhibitory inputs tend to arrive at the same time. We explore how the response of the output neuron - the firing rate and coherence with inhibitory oscillation - is modulated by jitter. Our main results are: (1) The mean number of inhibitory inputs determines whether variations in synchrony lead to changes in the neuron's sensitivity or whether they modulate the gain of the response (Sections 3.2, 3.3). (2) The response modulation by synchrony is most potent at gamma frequencies (Section 3.4). (3) Modulation of the single neuron response properties by inhibitory synchrony is consistent with the effects of attention observed in vivo [29,49] (Section 3.5). See Appendix A for a review of previous experimental results. Earlier reports of these results have appeared as abstracts [40,41].

\section{Methods}

\subsection{Modeling synchronous inhibitory inputs}

In a previously studied model of inhibitory interneurons connected by chemical synapses, the network produced oscillatory activity that consisted of a sequence of synchronized spike volleys $[23,75]$. First, we describe the statistics of the output of the interneuron network. Each spike volley was characterized by the number of spikes in the volley $a_{\mathrm{IV}}^{i}$ (with $i$ the volley index, $a$ the activity and IV indicating inhibitory volley), their mean spike time $t_{\mathrm{IV}}^{i}$ and their spike-time dispersion $\sigma_{\mathrm{IV}}^{i}$. There was variability in the volleys:

- The number of spikes varied across cycles. We used Poisson statistics, hence, the mean of $a_{\mathrm{IV}}^{i}$ across cycles was $a_{\mathrm{IV}}$ and its standard deviation was $\sqrt{a_{\mathrm{IV}}}$.

- The network oscillations were not perfectly regular. There was a stochastic drift in the interval between the arrival of consecutive volleys. The interval, $P_{i} \equiv t_{\mathrm{IV}}^{i+1}-$ $t_{\mathrm{IV}}^{i}$, was approximately normally distributed with mean (the period) $P$ and a coefficient of variation equal to $\mathrm{CV}_{T}$ (we use $C V_{T}$ rather than $C V_{P}$ to avoid confusion with a previously introduced synchrony measure [79]). The oscillation frequency was $f_{\text {osc }}=1 / P$.

- The mean of $\sigma_{\mathrm{IV}}^{i}$ across cycles was $\sigma_{\mathrm{IV}}$.

The spike-time dispersion, $\sigma_{\mathrm{IV}}$, is inversely related to the synchrony of the network oscillation. A synchronized network has $\sigma_{\mathrm{IV}}=1 \mathrm{~ms}(1 \mathrm{~ms}$ is the order of magnitude of the jitter caused by intrinsic noise in cortical slices [46]), whereas, for gamma oscillations, $\sigma_{\mathrm{IV}}=10 \mathrm{~ms}$ corresponds to an asynchronous network [75]. Hence, in the simulations of the model, $\sigma_{\mathrm{IV}}$ was varied between 1 and $10 \mathrm{~ms}$. The mean number of spikes per volley, $a_{\mathrm{IV}}$, is determined by the fraction of network neurons that is active on a given cycle, the size of the network, and the cortical GABAergic presynaptic release probability [42]. The network of interneurons was not explicitly simulated; rather the above statistics were used to model input spike trains representing the synchronous inhibitory input, as described below. These input spike trains will be referred to as 'network activity' throughout.

The method used to obtain synchronous volleys is illustrated in Fig. 1. First, a set of volley times $t_{\mathrm{IV}}^{i}$ was generated (with mean intervolley interval $P$ and a coefficient of variation $\mathrm{CV}_{T}$ ). Next, a binned spike-time probability (STP) was obtained by convolving the volley times with a Gaussian filter with standard deviation $\sigma_{\mathrm{IV}}$ and area $a_{\mathrm{IV}} \Delta t$ (the bin width $\Delta t=0.01 \mathrm{~ms}$ was equal to the integration timestep used in the simulations). The Gaussian filter was $40 \mathrm{~ms}$ long in order to accommodate at least 2 standard deviations for the maximum $\sigma_{\mathrm{IV}}$ used in the simulations. The peak of the Gaussian was located at the center of the filter at $20 \mathrm{~ms}$. As a result, the effect of changing $\sigma_{\mathrm{IV}}$ during the simulation acts with a delay of $20 \mathrm{~ms}$. Input spike times were generated as a Poisson process from the STP, as in [74]. Each input spike produced an exponentially decaying conductance pulse, $\Delta g_{\text {inh }} \exp \left(-t / \tau_{\text {inh }}\right)$ in the postsynaptic cell (inh $=$ inhibitory), yielding a current $I_{\text {syn }}=$ $\Delta g_{\text {inh }} \exp \left(-t / \tau_{\text {inh }}\right)\left(V-E_{\mathrm{GABA}}\right)$. In this expression $t$ is the time since the last presynaptic spike, $\tau_{\text {inh }}$ is a decay time 


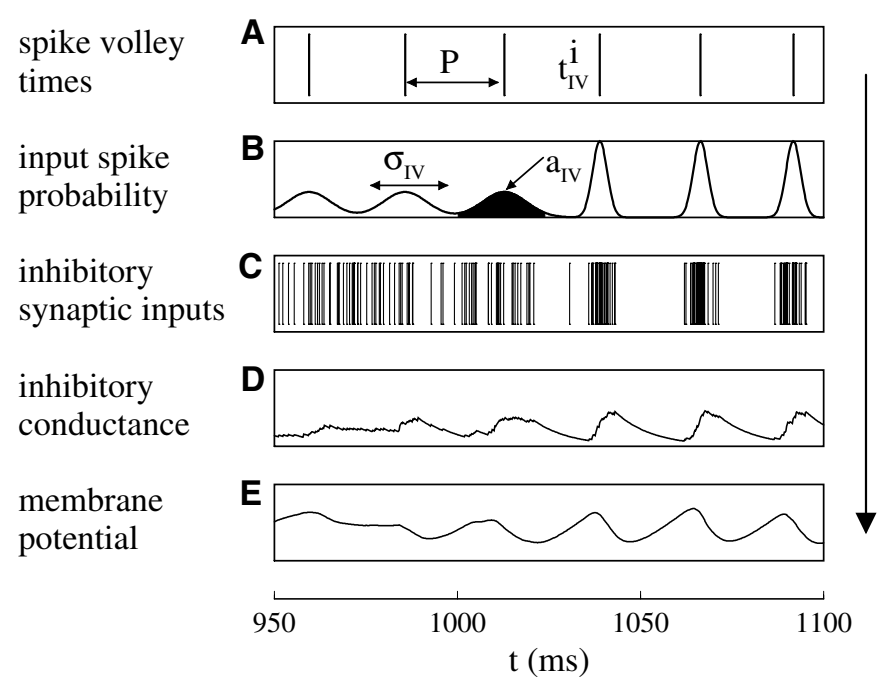

Fig. 1. Model for generating synchronous spike volleys in a simulated population of interneurons. (A) Spike volleys arrived at a rate of $f_{\text {osc }}$ volleys per second (the mean time separation between volleys was the period $P=1 / f_{\text {osc }}$ ). (B) Spike-time probability was generated by convolving spike volley times with a Gaussian filter; its width was $\sigma_{\mathrm{IV}}$ and its area (sum of the bins) was $a_{\mathrm{IV}} \Delta t$. (C) Spike times were generated as a Poisson process from the spike time probability. (D) Each input spike caused an exponentially decaying inhibitory conductance pulse with a unitary conductance $\Delta g_{\text {inh }}$ and a decay constant $\tau_{\text {inh. }}$. (E) Concomitant voltage fluctuations in the postsynaptic neuron.

constant, $\Delta g_{\text {inh }}$ is the unitary synaptic conductance, $V$ is the postsynaptic membrane potential, and $E_{\mathrm{GABA}}=-75 \mathrm{mV}$, is the reversal potential. The values of these parameters were varied and their specific values are given in each figure caption (see Appendix A). A similar procedure was used for synchronous excitatory inputs. The same notation holds with $E V$ (excitatory volleys) replacing $I V$ and exc (excitatory) replacing inh.

The resulting train of conductance pulses drove a single compartment neuron with Hodgkin-Huxley voltage-gated sodium and potassium channels, a passive leak current, synaptic currents as described above and a white noise current with mean $I$ and variance $2 D$. Full model equations are given in Appendix A [75]. They were integrated using a noise-adapted 2nd-order Runge-Kutta method [36], with time step $\mathrm{d} t=0.01 \mathrm{~ms}$. The accuracy of this integration method was checked for the dynamical equations without noise $(D=0)$ by varying $\mathrm{d} t$ and comparing the result to the one obtained with the standard 4th-order Runge-Kutta method [59] with a time-step $\mathrm{d} t$ of $0.05 \mathrm{~ms}$.

\subsection{Statistical analysis}

Simulations were run multiple times with different seeds for the random number generator, yielding different trials. Spike times $t_{i}^{j}$ (ith spike time during the $j$ th trial) of the target neuron were calculated as the time that the membrane potential crossed $0 \mathrm{mV}$ from below. The mean interspike interval, $\tau$, was calculated as the mean of all intervals during a given trial and then averaged across all trials. The mean firing rate $f$ was $1000 / \tau$ ( $\tau$ is in $\mathrm{ms}, f$ in $\mathrm{Hz}$ ). The coefficient of variation $(\mathrm{CV})$ was the standard deviation of the interspike intervals across one trial divided by their mean. The CV was then averaged across all trials. Errors in these statistics were estimated as the standard deviation across 10 equal-sized subsets of the data. The Fano factor (FF) was the variance of the spike count in a given time interval divided by the mean spike count.

For neurons receiving a synchronous inhibitory input, the firing rate versus current $(f-I)$ curves were obtained by systematically varying the depolarizing input current $I$ and calculating the firing rate as described above. $f-I$ curves were fitted to a sigmoidal function, $f(I)=\frac{A}{2}(1+$ $\left.\tanh \left(\lambda_{I}\left(I-\Delta_{I}\right)\right)\right)$. We also attempted to make the curves for different values of $\sigma_{\mathrm{IV}}$ overlap with a reference curve using the following three procedures: (1) A shift of $I$ over $\Delta_{I}$; (2) A multiplication of $I$ by $\lambda_{I}$; (3) A multiplication of $f$ by $\lambda_{f}$. The fitting procedures involved the MATLAB routine nonlinfit. Confidence intervals at $95 \%$ for the fitting parameters were obtained using the routine nlparci. Four different fits were used to minimize the difference between the fitted curve and the reference curve: (1) Shift of $I$; (2) Shift and scaling of $I$; (3) Shift of $I$ and scaling of $f$; (4) Shift of $I$ and scaling of $I$ and $f$. In most cases fit (3) yielded the best results or yielded results that were close to the best three-parameter fit (4). For the purpose of comparison we show only the results of fit (3).

The coherence of the output spike times with the underlying oscillations was determined using the spike-triggered average (STA) and the vector strength (VS). The local field potential (LFP) was estimated as the membrane potential of a model neuron receiving coherent synaptic inputs while being hyperpolarized to prevent action potentials. Spike times were obtained from another neuron receiving the same synaptic inputs. The STA was then calculated by taking the membrane potential from the first (hyperpolarized) neuron centered on the spike times of the second (depolarized) neuron [20]. The STA power spectrum was calculated using the MATLAB routine $p$ sd with standard windowing using a 2048 point fast Fourier transform (sampled at $0.2 \mathrm{~ms}$ ). The spike-field coherence (SFC) was computed as the STA power spectrum divided by the power spectrum of the LFP, as was done in the analysis of the experimental data [29].

The spike-phase coherence was determined from the variance and mean value of the phase of the spike time $t$ with respect to the oscillations. The phase was defined as $\phi=\left(t-t_{\mathrm{IV}}^{i}\right) /\left(t_{\mathrm{IV}}^{i+1}-t_{\mathrm{IV}}^{i}\right)$ [55]. Here $t_{\mathrm{IV}}^{i}$ is the last volley time before $t$ and $t_{\mathrm{IV}}^{i+1}$ is the first volley time after $t$. We determined the standard deviation $\sigma_{\phi}$ of $\phi$ and the VS [47],

$\mathrm{VS}=\sqrt{\langle\cos (2 \pi \phi)\rangle^{2}+\langle\sin (2 \pi \phi)\rangle^{2}}$.

Here $\langle\cdot\rangle$ is the average over all phases in a given trial and across all trials. VS is zero when $\phi$ is uniformly distributed between zero and one, and is one when the phase is constant. 


\section{Results}

\subsection{Inhibitory input synchrony modulates output firing rate and coherence}

The goal is to reproduce the effects of attention on cortical neurons in the visual cortex. In the model neuron that we simulated (Fig. 2) the stimulus-induced activation was represented by injecting an excitatory input current. In this baseline state, the object in the receptive field is not being attended. We explore the hypothesis that when attention is directed to an object in the neuron's receptive field, the discharge of the neuron is modulated through inhibitory inputs.

Fig. 2A shows the voltage response of a model neuron driven by an inhibitory input oscillating at approximately $40 \mathrm{~Hz}$. On each cycle of the oscillatory drive it received a volley with an average of $a_{\mathrm{IV}}=25$ pulses, each with a unitary peak conductance of $\Delta g_{\text {inh }}=0.044 \mathrm{mS} / \mathrm{cm}^{2}$. The time-

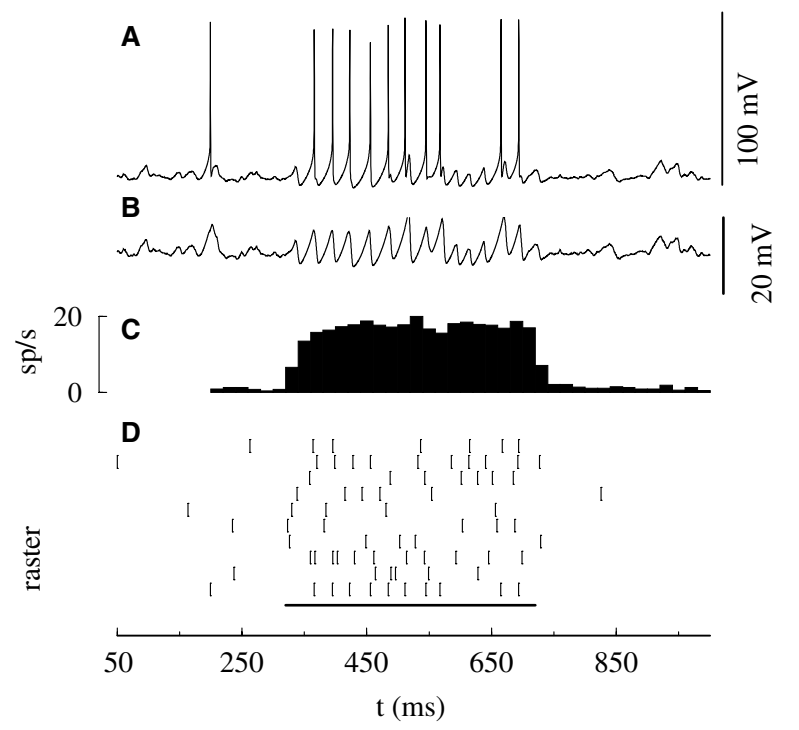

Fig. 2. Inhibitory input synchrony gated neural activity. (A) The membrane potential, (B) the local field potential (LFP), and (C) the firing rate as a function of time. (D) Rastergram of the first ten trials. During the time interval between $t=300$ and $700 \mathrm{~ms}$ (indicated by the bar in (D)), $\sigma_{\mathrm{IV}}$ was reduced to $2 \mathrm{~ms}$ from $8 \mathrm{~ms}$. The full parameter set is given in Appendix A. average of the inhibitory conductance was $0.44 \mathrm{mS} / \mathrm{cm}^{2}$, which was about four times larger than the leak conductance $g_{L}=0.1 \mathrm{mS} / \mathrm{cm}^{2}$. The neuron was not spontaneously active in the absence of synaptic inputs; hence, in order to make it spike in the presence of inhibition, a constant depolarizing current $I=4.0 \mu \mathrm{A} / \mathrm{cm}^{2}$ was also injected. The temporal dispersion of the input spike times was on average $\sigma_{\mathrm{IV}}$. In the baseline state, $\sigma_{\mathrm{IV}}=8 \mathrm{~ms}$, the inhibitory input was asynchronous, but during the time interval $t=300$ $\mathrm{ms}$ and $700 \mathrm{~ms}$ the input was made synchronous by decreasing $\sigma_{\mathrm{IV}}$ to $2 \mathrm{~ms}$. During the baseline state the neuron fired at a low average rate $(f=4.4 \pm 0.7 \mathrm{~Hz}$ obtained over a longer segment, $1000 \mathrm{~ms}$, than shown in the figure). The spiking statistics for this case and the figures below are summarized in Table 1.

When the inhibitory input was synchronous, the firing rate increased by a factor of four to $f=18.3 \pm 0.4 \mathrm{~Hz}$. This increase was robust across trials as indicated by the spike time histogram across 500 trials (Fig. 2C) and the rastergram for the first ten trials (Fig. 2D). Note that the timeaveraged inhibitory conductance remained constant during the entire trial, even during the episode of enhanced input synchrony. Thus, the increase in firing rate was due solely to the change in coherence.

In Fig. 2, a neuron was activated by a suitable stimulus that is ignored: initially, the neuron did not respond and the presence of the stimulus was not transmitted to downstream cortical areas, but during the time interval of increased input synchrony, the presence of the stimulus was signaled to downstream areas. The synchrony of the inhibitory input acted as gate. For the parameters of Fig. 2, when the stimulus was presented for a short interval, $400 \mathrm{~ms}$, the neuron did not produce a spike on most trials during the baseline state, but it did produce a couple of spikes during the period of increased input synchrony. The next three Sections focus on the statistical properties of the neuron's output spike train-how the firing rate and the coherence with the synaptic inputs varied with the parameters of the inhibitory input, specifically, $a_{\mathrm{IV}}$, $\sigma_{\text {IV }}$ and the oscillation frequency $f_{\text {osc }}$.

The spike trains of cortical neurons recorded in vivo are highly variable in time and across trials [67]. The spike trains obtained on different trials (see the rastergram in Fig. 2D) were significantly different from each other and

Table 1

Spiking statistics for Figs. 2 and 3

\begin{tabular}{|c|c|c|c|c|c|c|c|}
\hline$\sigma_{\mathrm{IV}}$ & $f(\mathrm{~Hz})$ & $\mathrm{CV}$ & $\mathrm{FF}$ & $\sigma_{\phi}$ & VS & $\operatorname{SFC}(\theta)$ & $\operatorname{SFC}(\gamma)$ \\
\hline \multicolumn{8}{|c|}{ Fig. 2} \\
\hline $8 \mathrm{~ms}$ & $4.40(0.67)$ & $0.961(0.137)$ & $1.204(0.189)$ & $0.189(0.029)$ & $0.710(0.045)$ & $0.30(0.58)$ & $0.14(0.14)$ \\
\hline $2 \mathrm{~ms}$ & $18.26(0.43)$ & $0.825(0.031)$ & $0.666(0.086)$ & $0.096(0.007)$ & $0.878(0.006)$ & $0.005(0.002)$ & $0.026(0.010)$ \\
\hline \multicolumn{8}{|c|}{ Fig. 3} \\
\hline $4 \mathrm{~ms}$ & $22.33(0.44)$ & $0.985(0.038)$ & $1.054(0.327)$ & $0.181(0.009)$ & $0.685(0.012)$ & $0.006(0.001)$ & $0.025(0.015)$ \\
\hline $2 \mathrm{~ms}$ & $34.65(0.49)$ & $0.781(0.022)$ & $0.646(0.158)$ & $0.148(0.007)$ & $0.744(0.004)$ & $0.002(0.001)$ & $0.038(0.022)$ \\
\hline
\end{tabular}

The firing rate $f$, coefficient of variation $\mathrm{CV}$, Fano factor $\mathrm{FF}$, phase standard deviation $\sigma_{\phi}$, vector strength VS, SFC in theta range (4.5-15 $\left.\mathrm{Hz}\right)$ and gamma range $(34-44 \mathrm{~Hz})$ were calculated as described in Methods. Errors are given between parentheses and are the standard deviation across 10 sets. 
the interspike intervals ranged from $5 \mathrm{~ms}$ to the $100 \mathrm{~s}$ of ms. The irregularity of spike trains in time was quantified by the coefficient of variation $(\mathrm{CV})$ : the standard deviation of the interspike intervals in time, divided by their mean. $\mathrm{CV}$ values between 0.5 and 1.0 are typical for in vivo recordings [67]. The values obtained for Fig. 2 were in this range, $\mathrm{CV}=0.96 \pm 0.14$ during the baseline state and $\mathrm{CV}=0.83 \pm 0.03$ during the interval with increased input synchrony. We used longer segments than shown in Fig. 2-1000 ms across 500 trials - to obtain more robust estimates for the $\mathrm{CV}$ values. In Fig. $2 \mathrm{~A}$ the spike train looked quite regular during the period with high input synchrony because it was entrained to the input oscillation. Although the interspike intervals were variable they were approximate multiples of the oscillation period. Hence, the high $\mathrm{CV}$ value was a consequence of the multimodality of the interspike interval distribution.

The irregularity of spike trains across trials is commonly expressed as the Fano factor (FF): the variance of the spike counts during a time interval divided by the mean. For a $1000 \mathrm{~ms}$ interval, $\mathrm{FF}=1.2 \pm 0.2$ (baseline) and $\mathrm{FF}=$ $0.67 \pm 0.09$ (increased input synchrony). In comparison, a homogeneous Poisson process has $\mathrm{CV}=1$ and $\mathrm{FF}=1$. The value of the Fano factor is a direct consequence of our choice of model parameters: there is variability across trials in the arrival time of the inhibitory volley, $\mathrm{CV}_{T}$, variability in the number and the timing of the inputs in the volley, and intrinsic noise. Consistent with this expectation, much smaller Fano factors were obtained when one or more of these sources of variability were absent.

The spike-field coherence (SFC) has been used to quantify the degree of coherence of spike trains with the local field potential (LFP). The LFP is measured using an extracellular electrode and is assumed to reflect the synaptic inputs to neurons close to the electrode and their resulting activity [30]. It is not known in general how many neurons contribute to the LFP and whether it is dominated by the activity of local neurons or whether it more closely reflects the synaptic activity due to presynaptic neurons. We calculated the LFP as the membrane potential fluctuations in a neuron that was hyperpolarized to prevent action potentials. The SFC in the gamma frequency range $(34-44 \mathrm{~Hz})$ decreased from $0.14 \pm 0.14$ in the baseline state to $0.026 \pm 0.010$ during the period of increased inhibitory input synchrony. This counterintuitive result underscores the fact that in modeling studies the SFC so defined may not be the best way of calculating the coherence; hence, the vector strength (VS) will be used here instead, although for comparison with experiments in Section 3.5 the SFC will be used. The relation between the SFC and the VS is explained in Appendix A. During the state of increased inhibitory input synchrony, the VS increased to $0.878 \pm 0.006$ from $0.710 \pm 0.045$ in the baseline state. The key constraint is that one needs enough spikes to accurately estimate the SFC. The SFC for the baseline state was recalculated, but with the injected current increased from $4.0 \mu \mathrm{A} / \mathrm{cm}^{2}$ to $6.0 \mu \mathrm{A} / \mathrm{cm}^{2}$ in order to make the neuron's

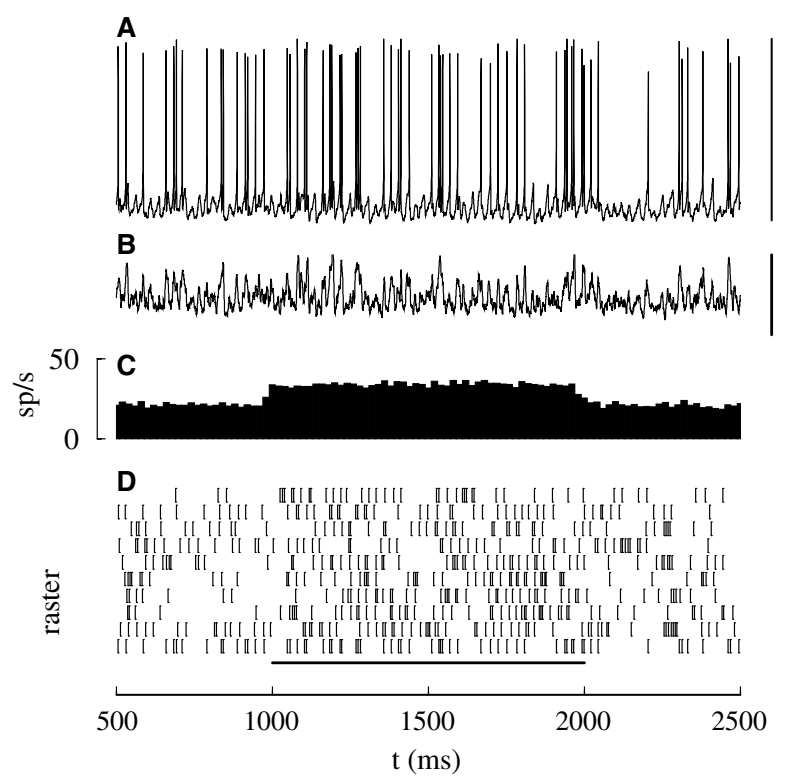

Fig. 3. The inhibitory input synchrony modulated the output firing rate. (A) The membrane potential, (B) the local field potential (LFP), and (C) the firing rate as a function of time. (D) Rastergram of the first ten trials. During the time interval between $t=1000$ and $2000 \mathrm{~ms}$ (indicated by the bar in (D)), $\sigma_{\mathrm{IV}}$ was reduced to $2 \mathrm{~ms}$ from $4 \mathrm{~ms}$. The full parameter set is given in Appendix A.

firing rate similar to that for synchronous inputs. The SFC was $0.008 \pm 0.007$ in the gamma range and $0.016 \pm$ 0.012 in the theta range.

Fig. 3 shows another example of firing rate modulation with input synchrony. The model neuron was again driven by a synchronous inhibitory drive, but part of the depolarization was provided by a homogeneous excitatory Poisson process. During the time interval between $t=1000$ and $2000 \mathrm{~ms}, \sigma_{\mathrm{IV}}$ was decreased from $4 \mathrm{~ms}$ to $2 \mathrm{~ms}$. For $t<$ $1000 \mathrm{~ms}$, the firing rate was $22.3 \mathrm{~Hz}$ and $\mathrm{VS}=0.685 \pm$ 0.012 (other statistics are listed in Table 1). When the input synchrony was increased the firing rate increased to 34.6 $\mathrm{Hz}$ and $\mathrm{VS}=0.744 \pm 0.004$. In contrast to Fig. 2, the spike trains looked more like the ones found in vivo. The degree of synchrony also modulated the firing rate at higher values (Fig. 3), rather than just acting as a gate (Fig. 2).

In both examples the model predicts that synchronyinduced increases in firing rate are associated with a decrease in firing variability and an increase in coherence.

\subsection{Modulation of $f-I$ curves with input synchrony}

The firing rate was plotted as a function of the depolarizing current $I$ and the jitter $\sigma_{\mathrm{IV}}$ in (Fig. 4A). The firing rate ranged from 0 to $80 \mathrm{~Hz}$ for $1 \mathrm{~ms} \leqslant \sigma_{\mathrm{IV}} \leqslant 6 \mathrm{~ms}$ and $2 \mu \mathrm{A} /$ $\mathrm{cm}^{2} \leqslant I \leqslant 7.5 \mu \mathrm{A} / \mathrm{cm}^{2}$. An increase in $\sigma_{\mathrm{IV}}$ usually led to a reduction in firing rate (dashed arrow in Fig. 4A). The $f-I$ curve for constant $\sigma_{\mathrm{IV}}$ had a knee for small values of $\sigma_{\mathrm{IV}}$ (arrow in Fig. 4A). At this point in the neuron's operating range, decreasing $\sigma_{\mathrm{IV}}$ (increasing input synchrony) did not 

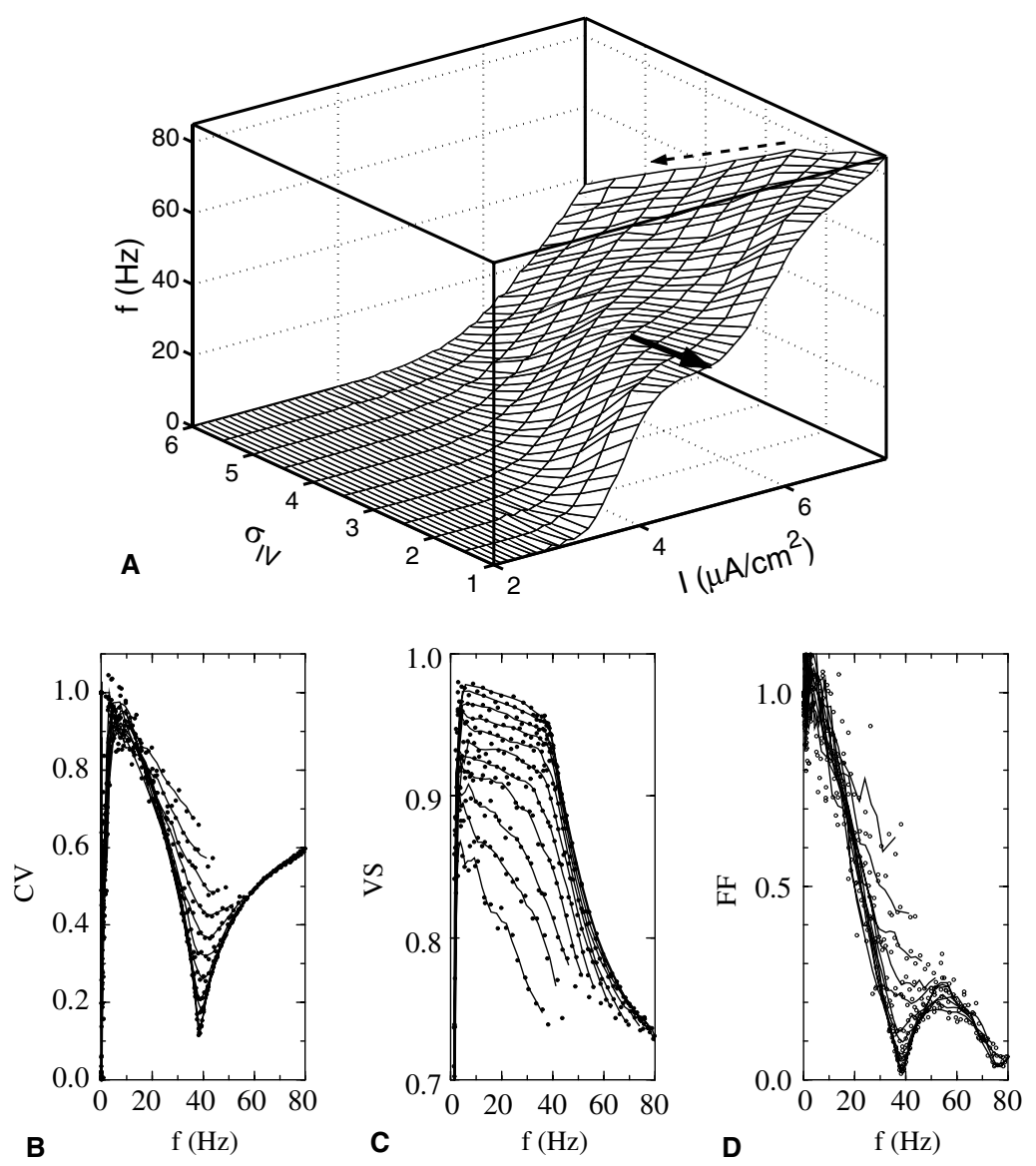

Fig. 4. The firing rate and the coherence for $a_{\mathrm{IV}}=50$. (A) Firing rate $f$ as a function of $\sigma_{\mathrm{IV}}$ and $I$. (B) Coefficient of variation, (C) Vector strength, and (D) Fano factor as a function of firing rate. The solid lines in (B, D) are 3-point running averages for, from bottom to top, $\sigma_{\mathrm{IV}}=1,2, \ldots, 5 \mathrm{~ms}$, circles are the data points. The same $\sigma_{\mathrm{IV}}$ values are shown in (C), but now ordered from top to bottom.

lead to an increase in firing rate, but the coherence did increase (see below). Neither did an increase in driving current result in a higher firing rate. Because of the stochastic nature of the dynamics the firing rate could be arbitrarily low. For practical purposes the onset of firing was defined as the point where the firing rate exceeded $1 \mathrm{~Hz}$. This means that on average more than one spike should be observed on $1 \mathrm{~s}$ long trials. The current value at which this happened increased steadily with increasing $\sigma_{\text {IV }}$ (Fig. 4A).

To determine how the variability and the coherence were related to the firing rate $f, \mathrm{CV}$-f and VS-f plots were made at constant $\sigma_{\text {IV }}$ values (Fig. 4B,C). Each data point represented a point on the $f$ versus $I$ and $\sigma_{\mathrm{IV}}$ surface. The $\mathrm{CV}$ was 1 for small firing rates, but decreased with $f$ until $f \approx 40 \mathrm{~Hz}$, at which point the $\mathrm{CV}$ increased with $f$ again. The minimum $\mathrm{CV}$ value reached at $f \approx 40 \mathrm{~Hz}$ was $\mathrm{CV} \approx 0.1$ for $\sigma_{\mathrm{IV}}=1 \mathrm{~ms}$ and increased with $\sigma_{\mathrm{IV}}$. The relationship between the knee in the $f-I$ curve and the dip in the CV- $f$ curve can be understood in terms of phase locking to the synchronous inhibitory synaptic drive. When the neuron is phase-locked it fires one spike on each cycle and the firing rate is $40 \mathrm{~Hz}$. The interspike interval is approximately equal to duration of an oscillation cycle, but there is some variability due to jitter of the spike time within each cycle. The CV is small for this situation. For smaller driving currents, the phase locking becomes less stable and the firing rate falls below $40 \mathrm{~Hz}$. The neuron will then skip cycles leading to a bimodal distribution of interspike intervals: the intervals are either approximately equal to one cycle or two cycles. As a result the CV increases sharply. For larger driving currents phase locking also becomes unstable, but now the firing rate exceeds $40 \mathrm{~Hz}$. On some cycles there are more than one spike, yielding a bimodal interspike interval distribution. The $\mathrm{CV}$ also increases sharply for this situation. A more detailed description of phase locking to a periodic inhibitory drive can be found in Ref. [74].

The most regular spike trains were obtained near the knee in the firing rate surface. The VS had its highest value for small firing rates and decreased very slowly with firing rate up to $f \approx 40 \mathrm{~Hz}$. For $f>40 \mathrm{~Hz}$, there was a precipitous drop in VS with firing rate. This occurred because for $f<40 \mathrm{~Hz}$ the neuron produced at most one spike per cycle at approximately the same phase with respect to the oscillation-hence the VS was high, but for $f>40 \mathrm{~Hz}$, there could be two spikes on a given cycle, at two different phases, and hence the VS was lower. The behavior of the FF was similar to that of the CV (Fig. 4D). 
We performed the same analysis on a model neuron with the parameters used to generate the spike trains in Fig. 3. The firing rate surface is shown in Fig. 5A. There was no 'knee'; instead, the firing rate always decreased with increasing $\sigma_{\mathrm{IV}}$ and always increased with $I$. The variability of the spike trains (Fig. 5B) was highest for low firing rates and the $\mathrm{CV}$ took values ranging between $\mathrm{CV}=0.9$ and 1.3 . The CV remained constant for the highest $\sigma_{\mathrm{IV}}=7 \mathrm{~ms}$ to $10 \mathrm{~ms}$ values studied, but decreased with firing rate for lower $\sigma_{\mathrm{IV}}$ values. There was no evidence for a minimum at $f \approx 40 \mathrm{~Hz}$, in contrast to the preceding case. The VS decreased linearly as a function of firing rate (Fig. 5C) and decreased in all cases with $\sigma_{\mathrm{IV}}$. The behavior of the FF was similar to that of the CV (Fig. 5D).

The simulations for which part of the depolarizing drive is provided by excitatory synaptic inputs are more realistic. However, when the rate of the excitatory inputs is varied, both the mean as well as the variance of the current fluctuations are altered (see, for instance, Ref. [76]). This makes it harder to distinguish changes due to the mean driving current from those due to the variance in the driving current. For this reason, the following analysis is only performed on neurons driven by a depolarizing current.

The shape of the $f-I$ curves varied with $\sigma_{\mathrm{IV}}$ for $a_{\mathrm{IV}}=10$ and $a_{\mathrm{IV}}=50$ (Fig. 6A, B). For $a_{\mathrm{IV}}=10$, the $f-I$ curves were non-saturating (Fig. 6A), but could not be fitted by a power law relationship between $f$ and $I$. In previous studies it was found that the sensitivity and the gain of $f-I$ curves were modulated by the statistics of the synaptic input $[26,52]$. Therefore we tried to collapse the $f-I$ curves for different $\sigma_{\mathrm{IV}}$ onto a reference curve, here taken to be $\sigma_{\mathrm{IV}}=$ $1 \mathrm{~ms}$, by rescaling the firing rate (gain change: $f \rightarrow f\left(\lambda_{f}\right.$ ) and changing the sensitivity (shift in $I, I \rightarrow I-\Delta_{I}$ ). The fitting parameters, $\lambda_{f}$ and $\Delta_{I}$, were plotted as a function of $\sigma_{\mathrm{IV}}$ in Fig. 6C (for $\sigma_{\mathrm{IV}}=1 \mathrm{~ms}$, by definition, $\lambda_{f}=1$ and $\left.\Delta_{I}=0\right)$. For $a_{\mathrm{IV}}=50$, the $f-I$ curves saturated at approximately $40 \mathrm{~Hz}$ (Fig. 6B), which corresponded to the knee in Fig. 4. It was not possible to scale the curves by $\lambda_{f}$ in order to make them collapse since that would alter the saturation value. For $\sigma_{\mathrm{IV}} \leqslant 4 \mathrm{~ms}$, the $f-I$ curves were well fitted by a sigmoid function, $f=A / 2\left(1+\tanh \left(\lambda_{I}\left(I-\Delta_{I}\right)\right)\right)$. The fitting parameter $\Delta_{I}$ corresponded again to a shift in sensitivity, $\lambda_{I}$ corresponded to the slope of the sigmoid and $A$ was the saturation value of the firing rate. The higher the value of $\lambda_{I}$ the smaller the range of current values over which the firing rate went from firing rates near zero to $f \approx A$. For firing rates $f \ll A / 2$, this can be interpreted as a change in gain, with the gain proportional to $\lambda_{I}$. We performed the fitting procedure with $A$ as a free parameter, but this did not result in a better fit compared with the fit obtained by setting $A$ equal to $f_{\text {osc }}$. The fitting parameters $\Delta_{I}$ and $\lambda_{I}$ are plotted versus $\sigma_{\mathrm{IV}}$ in Fig. $6 \mathrm{D}$. Increasing $\sigma_{\mathrm{IV}}$ led to
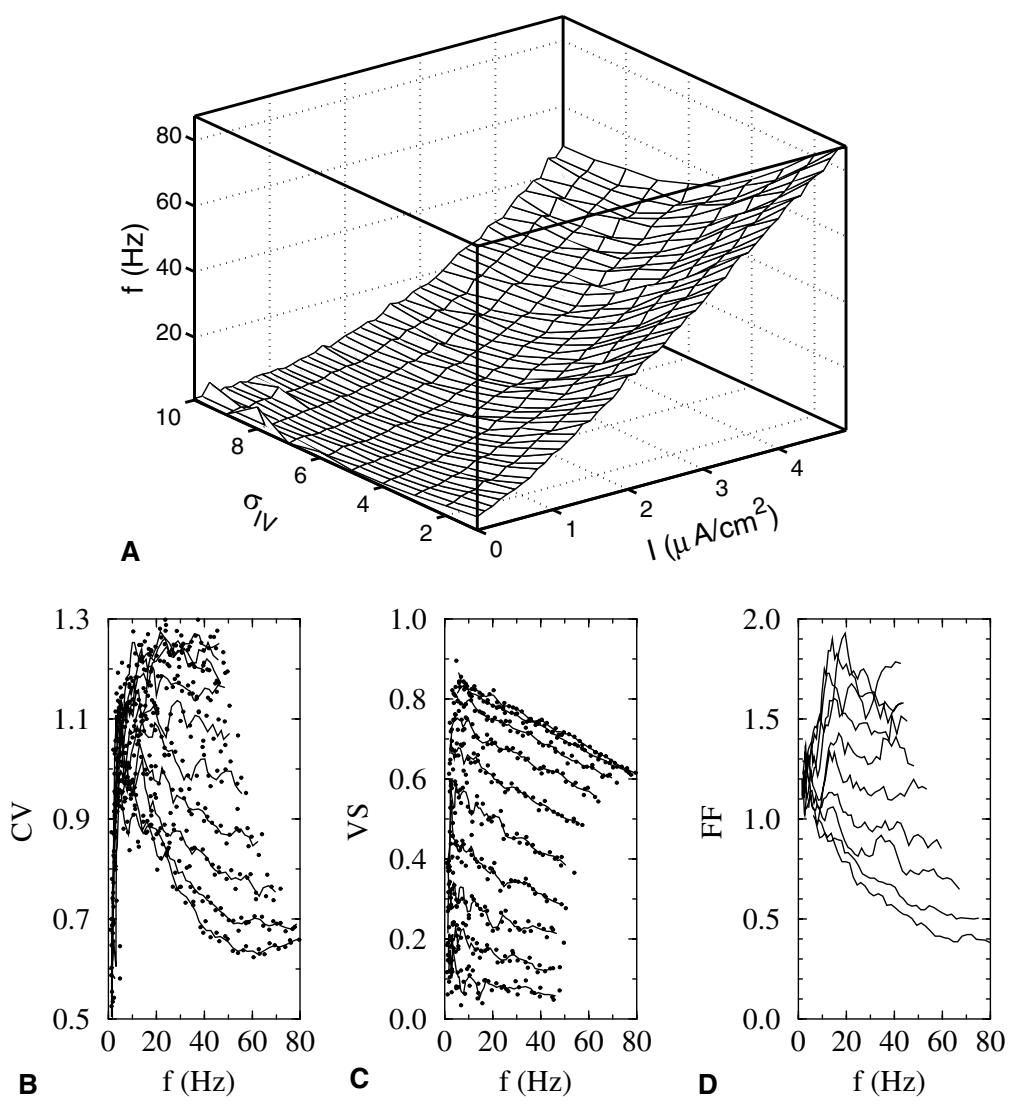

Fig. 5. The firing rate and the coherence for $a_{\mathrm{IV}}=10$ together with a background excitatory synaptic input. Panels are as in Fig. 4, parameters are as in Fig. 3. For clarity, we show only the 3-point running average in panel $\mathrm{D}$. 

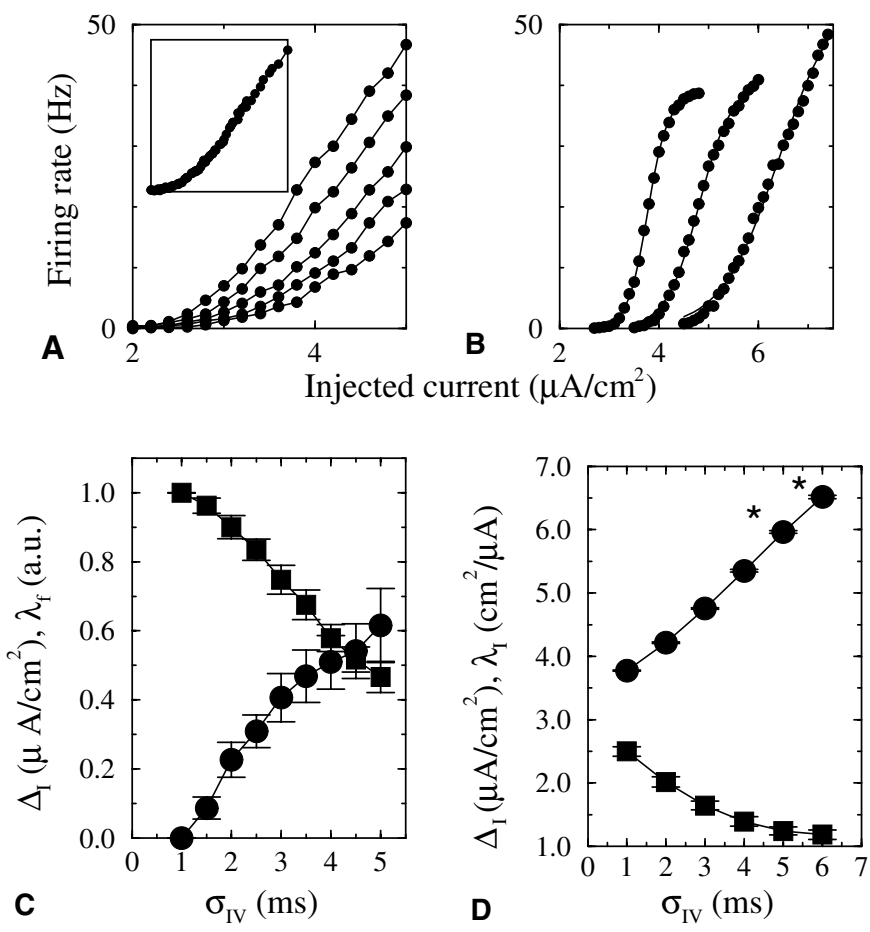

Fig. 6. Subtractive and divisive scaling of $f-I$ curves with inhibitory synchrony in the computer model. (A) Multiplicative gain modulation with inhibitory synchrony. $a_{\mathrm{IV}}=10$, from top to bottom $\sigma_{\mathrm{IV}}=1,2,3,4$ and $5 \mathrm{~ms}$. Inset: all curves could be collapsed by a shift in the current and a rescaling of the firing rate axis. (B) Shift in neural sensitivity with inhibitory synchrony, $a_{\mathrm{IV}}=50$, from left to right, $\sigma_{\mathrm{IV}}=1,3$, and $5 \mathrm{~ms}$. The solid lines are fits to a sigmoid function, filled circles are the simulation results. (C, D) Fitting parameters as a function of $\sigma_{\mathrm{IV}}$. (C) The shift $\Delta_{I}$ (circles) and firing rate gain $\lambda_{f}$ (squares) necessary to make the curves in (A) collapse on the $\sigma_{\mathrm{IV}}=1$ reference curve. (D) The midpoint $\Delta_{I}$ (circles) and slope $\lambda_{I}$ (squares) of the best-fitting sigmoid. The asterisk labels $f-I$ curves that were not well fitted by a sigmoid.

a shift to the right $\left(\Delta_{I}>0\right)$, decreasing sensitivity and stretching the sigmoid $\left(\lambda_{I}\right.$ decreased), yielding a larger dynamical range.

The shift in sensitivity going from $\sigma_{\mathrm{IV}}=5 \mathrm{~ms}$ to $\sigma_{\mathrm{IV}}=$ $1 \mathrm{~ms}$ was much higher for $a_{\mathrm{IV}}=50$ than for $a_{\mathrm{IV}}=10$. In contrast, for $a_{\mathrm{IV}}=10$, the gain change was more pronounced than for $a_{\mathrm{IV}}=50$ and it also extended over a larger range. The difference between $a_{\mathrm{IV}}=10$ and $a_{\mathrm{IV}}=50$ persisted when part of the depolarizing current was provided by excitatory synaptic inputs. We determined the $f-I$ curves for $a_{\mathrm{IV}}=10$ (Fig. 7A) and $a_{\mathrm{IV}}=50$ (Fig. 7B) while the neuron was driven by varying amounts of excitatory synaptic inputs $\left(\sigma_{\mathrm{IV}}\right.$ was kept fixed at $\left.1 \mathrm{~ms}\right)$. There was no knee in the $f-I$ curve for $a_{\mathrm{IV}}=10$. In contrast, there was a knee for $a_{\mathrm{IV}}=50$ that persisted for input rates up 700 EPSPs per second. The knee was accompanied by a dip in the CV versus $I$ curve (Fig. 7D). For $a_{\mathrm{IV}}=10$ (Fig. 7C), the dip was much less pronounced or absent.

In summary, for small $a_{\mathrm{IV}}$, changes in gain with input synchrony dominated, whereas for large $a_{\mathrm{IV}}$, changes in sensitivity dominated. The mean number of inputs on each oscillation cycle, $a_{\mathrm{IV}}$, was an important characteristic of the
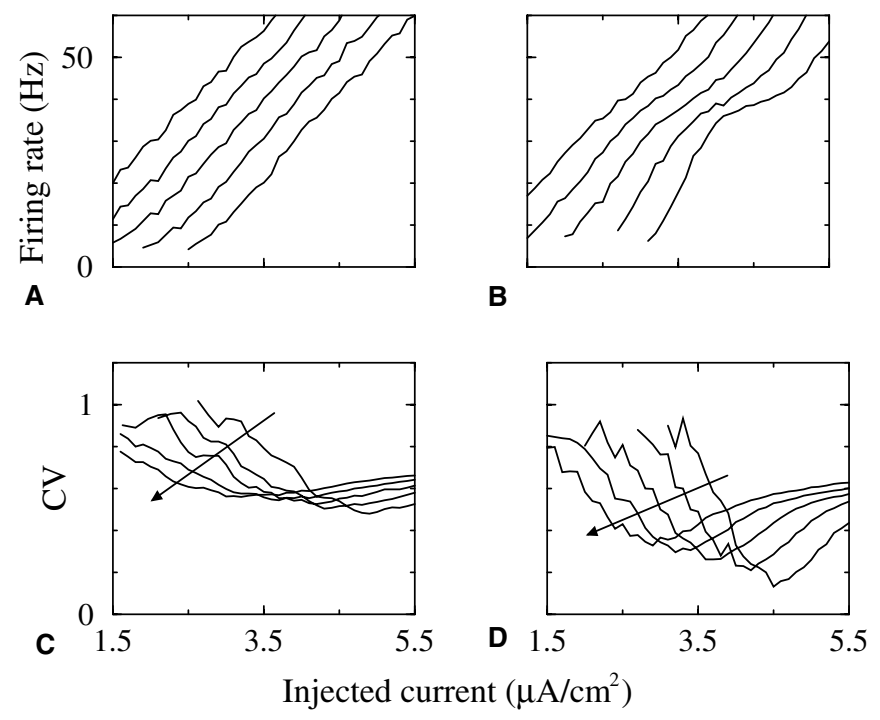

Fig. 7. The difference between $f-I$ curves for $a_{\mathrm{IV}}=10$ and 50 is robust against excitatory Poisson spike train inputs. The (A, B) firing rate and (C, D) coefficient of variation is plotted versus current for (A, C) $a_{\mathrm{IV}}=10$ and (B, D) $a_{\mathrm{IV}}=50$. The jitter is $\sigma_{\mathrm{IV}}=1 \mathrm{~ms}$. The input rate was 100,300 , 500, 700 and 900 EPSPs per second increasing from right to left (A, B) or as indicated by the direction of the arrow $(\mathrm{C}, \mathrm{D})$.

presynaptic network and could be modulated (see Section $4)$.

\subsection{Shift in sensitivity versus change of gain}

The responses of neurons driven by excitatory and inhibitory synaptic inputs have been studied extensively (see Section 4). A change in the mean conductance of the synaptic inputs results in a shift of the $f-I$ curve, whereas a change in the variance of the conductance corresponds to a change in gain $[14,26]$. Is it possible to understand the results of the preceding section in terms of the 'mean' and the 'variance'?

The mean and variance of the conductance can be calculated either by taking one long trial and performing a time average or averaging an observation of the variable at one specific time across many trials. For a stationary process, for which the statistics do not change in time, these two methods are equivalent. The time-averaged conductance of the synchronous inhibitory input does not depend on the jitter, $\sigma_{\mathrm{IV}}$, but the neuron's response does. Hence, one would infer that the change in firing rate with $\sigma_{\text {IV }}$ would be due solely to the 'variance'.

In Fig. 8Aa, dashed line, we show the inhibitory conductance as a function of time on a given trial. There was a peak every $25 \mathrm{~ms}$, but its amplitude was different on each cycle because the number of inputs and their timing differed across cycles. However, the conductance waveform averaged across trials (Fig. 8Aa, solid line) was the same on each cycle. Furthermore, the conductance waveform obtained on one cycle by averaging across all cycles in one trial (Fig. 8Ab), was the same as the trial average. The variability across trials was also the same as the 

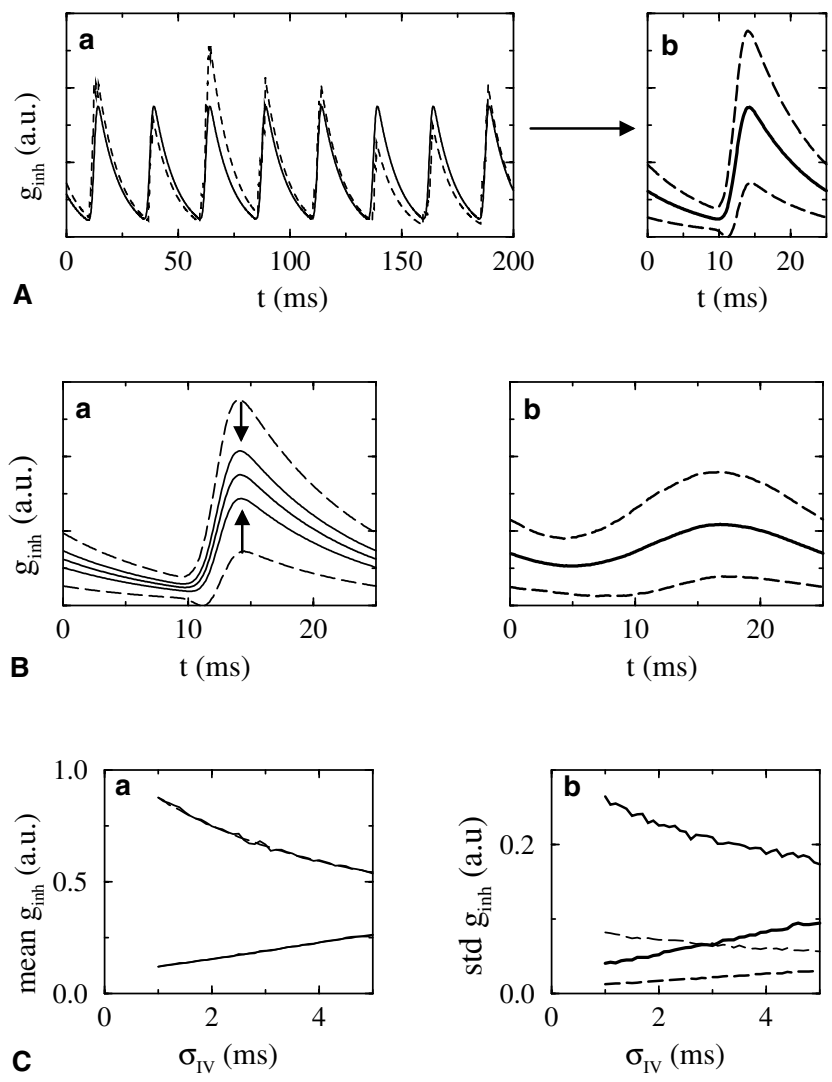

Fig. 8. Variability of the inhibitory conductance depends on the synchrony $\sigma_{\mathrm{IV}}$ and the mean number of inputs $a_{\mathrm{IV}}$. (Aa) Inhibitory conductance as a function of time for $a_{\mathrm{IV}}=10$ and $\sigma_{\mathrm{IV}}=1 \mathrm{~ms}$, we plot the mean across trials (solid line) and a sample trace for one trial (dashed line). (Ab) The average over cycles (solid line) for one trial is the same as the average of one cycle across multiple trials. The dashed curves are the mean plus or minus twice the standard deviation across trials. (Ba) Increasing the mean number of inputs per cycle to $a_{\mathrm{IV}}=100$ (thick solid line) from $a_{\mathrm{IV}}=10$ (dashed line) decreases the standard deviation in the conductance (arrows). (Bb) Decreasing the degree of input synchrony to $\sigma_{\mathrm{IV}}=5 \mathrm{~ms}$ reduces the temporal modulation of the conductance. (Ca) The maximum (top) and minimum (bottom) of the mean across cycles of the conductance waveform as a function of $\sigma_{\mathrm{IV}}$. The mean did not depend on the value of $a_{\mathrm{IV}}(\mathrm{Cb})$ The standard deviation of the conductance values across cycles at the phase when the maximum (top) or minimum (bottom) value of the mean is reached. Data is for $a_{\mathrm{IV}}=10$ (solid line) and $a_{\mathrm{IV}}=100$ (dashed line).

variability across cycles and is visualized in Fig. $8 \mathrm{Ab}$ as the mean plus or minus twice the standard deviation. In the following we refer to either the mean or variability without specifying whether it is across trials or cycles. We also kept the time-averaged mean conductance constant while varying $a_{\mathrm{IV}}$ by scaling the unitary conductance as $1 / a_{\mathrm{IV}}$. The mean conductance waveform did not depend on $a_{\mathrm{IV}}$, but the variability decreased with increasing $a_{\mathrm{IV}}$ (Fig. 8Ba). The mean conductance waveform as well as the variability depended on $\sigma_{\mathrm{IV}}$ (Fig. 8Bb).

When a neuron is driven by a time-varying inhibitory conductance and the injected current is in an appropriate range, it can only spike when the inhibitory conductance is small. There is a value for the inhibitory conductance above which the neuron will not spike. This value plays a role similar to the voltage threshold for an action potential in the integrate-and-fire neuron. The minimum value reached in the mean conductance waveform can thus be identified with the 'mean', since it determines the distance to threshold, and the variance in its value across cycles as the 'variance'. The maximum and minimum value of the mean conductance waveform reached during an oscillation cycle is plotted as a function of $\sigma_{\mathrm{IV}}$ in Fig. $8 \mathrm{Ca}$. The minimum increased and the maximum decreased as a function of $\sigma_{\mathrm{IV}}$. The variance in the conductance at the time its mean has a minimum increased with $\sigma_{\mathrm{IV}}$ (Fig. $8 \mathrm{Cb}$ ). The rate of increase with $\sigma_{\mathrm{IV}}$ was higher for smaller values of $a_{\mathrm{IV}}$. For large $a_{\mathrm{IV}}$, the 'variance' was small and an increase in $\sigma_{\mathrm{IV}}$ would increase the 'mean'. The corresponding effect was a shift of the $f-I$ curve. Another consequence of having a small 'variance' is that the neuron became entrained, leading to saturation of the firing rate. Increasing $\sigma_{\mathrm{IV}}$, for small $a_{\mathrm{IV}}$, both increased the 'variance' and the 'mean', hence there was both a shift as well as a change of gain in the $f-I$ curve. This analysis indicates that for time-varying conductances the 'variance' corresponds to the variability of the conductance waveform across trials and that this variability could modulate the gain of the neuron's response.

\subsection{Attentional modulation of $f-I$ curves is maximal for gamma frequency oscillations}

The strength of the modulation by synchrony was determined by the extent to which it could alter a neuron's firing rate. This change was quantified in terms of the ratio of the firing rate for moderate synchrony $\left(\sigma_{\mathrm{IV}}=4 \mathrm{~ms}\right)$ over the firing rate for weak synchrony $\left(\sigma_{\mathrm{IV}}=10 \mathrm{~ms}\right)$ because it reflected the strength of multiplicative interactions. This quantity had a peak at approximately $40 \mathrm{~Hz}$ (Fig. 9). The resonance peak was mainly determined by the time constant of inhibition, $\tau_{\text {inh }}$. When $\tau_{\text {inh }}$ was increased the peak shifted to the left, and when $\tau_{\text {inh }}$ was decreased, the peak shifted to the right (data not shown). We studied how robust the resonance was. When the driving current was increased from 5 to $6 \mu \mathrm{A} / \mathrm{cm}^{2}$ or when the number of pulses per cycle was increased from $a_{\mathrm{IV}}=25$ to 250 , the resonance

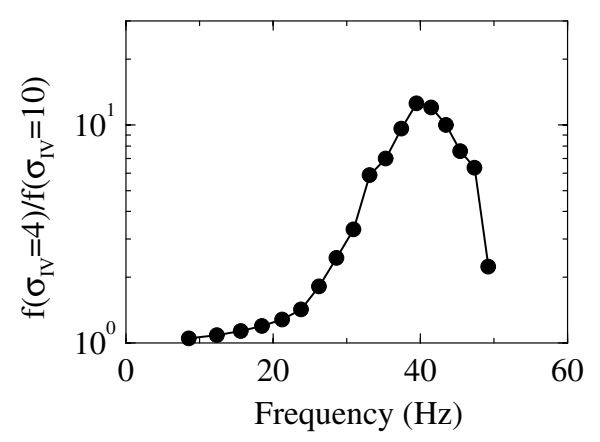

Fig. 9. Gamma-frequency-range resonance in the strength of attentional modulation of the firing rate. Strength of attentional modulation is quantified as the ratio of the firing rate for $\sigma_{\mathrm{IV}}=4 \mathrm{~ms}$ over that for $\sigma_{\mathrm{IV}}=10 \mathrm{~ms}$. The ratio attains its maximal value at approximately $40 \mathrm{~Hz}$. 
disappeared. This indicates that for the resonance to occur the neuron needs to be subthreshold and there should be sufficient variability in the inhibitory inputs. The effect was moderately robust against jitter in the spike volley times: We obtained resonance peaks for $\mathrm{CV}_{T}$ values up to approximately 0.05 .

\subsection{Modeling the experimental data}

Three key papers $[29,49,64]$ reported experimental results on attentional modulation of the firing rate and the coherence of V4 neurons in macaques (see Appendix A). In these papers, a stimulus was presented either inside or outside the receptive field of a neuron, and the focus of attention of the animal was either directed away from or into the receptive field by appropriate cues. The neurons that were recorded from were often orientation selective. We show here that the effects of attention in these studies can be explained by changes in the synchrony of local interneuron networks. As mentioned before, the network activity was not explicitly simulated, but instead the effects of modulating the synchrony on the principal neuron were modeled by dynamically changing $\sigma_{\mathrm{IV}}$ during the trial.

The effects of attention were modeled by changing the parameters that control the synchronous inhibitory drive. In the attended state, input synchrony was increased by reducing the value of $\sigma_{\mathrm{IV}}$ when compared with the nonattended state. The receptive field and orientation selectivity were modeled as a constant depolarizing current $I$ or by an excitatory Poisson process. We made the cell orientation-selective $[27,68]$ by changing the amount of current injected into the neuron as a function of the stimulus orientation $\psi: I_{A}=I_{O}+A_{O} \exp \left(-\psi^{2} / 2 \sigma_{\psi}^{2}\right)$, where $I_{O}$ is the baseline current in the presence of a stimulus, $A_{O}$ is the strength of orientation selectivity, and $\sigma_{\psi}$ represents the degree of selectivity.

Our aim was to show that modulating inhibitory synchrony can account for the experimentally reported effects of attention. Similar results were obtained for different sets of parameters. The experimental results were from different cortical areas and different conditions, and are not in complete agreement, so one unique set of parameters is unlikely to account for all experiments. The different regimes needed to model the data from the different laboratories may provide insight into the state of the cortex in the different conditions.

\subsubsection{Computer Experiment I: Fig. 4 of McAdams and Maunsell [49]}

The effects of attention on the orientation-tuning curve were modeled as a decrease in the spike-time dispersion from $\sigma_{\mathrm{IV}}=8$ to $7 \mathrm{~ms}$. The firing rate in the non-preferred orientation increased minimally from $4.5 \mathrm{~Hz}$ to $5.5 \mathrm{~Hz}$ in the attended state, whereas the firing rate in the preferred orientation increased by $20 \%$ from $14.2 \mathrm{~Hz}$ to $17.1 \mathrm{~Hz}$ (Fig. 10Aa), compatible with the experimental data. The asymptote was subtracted from the orientation-tuning
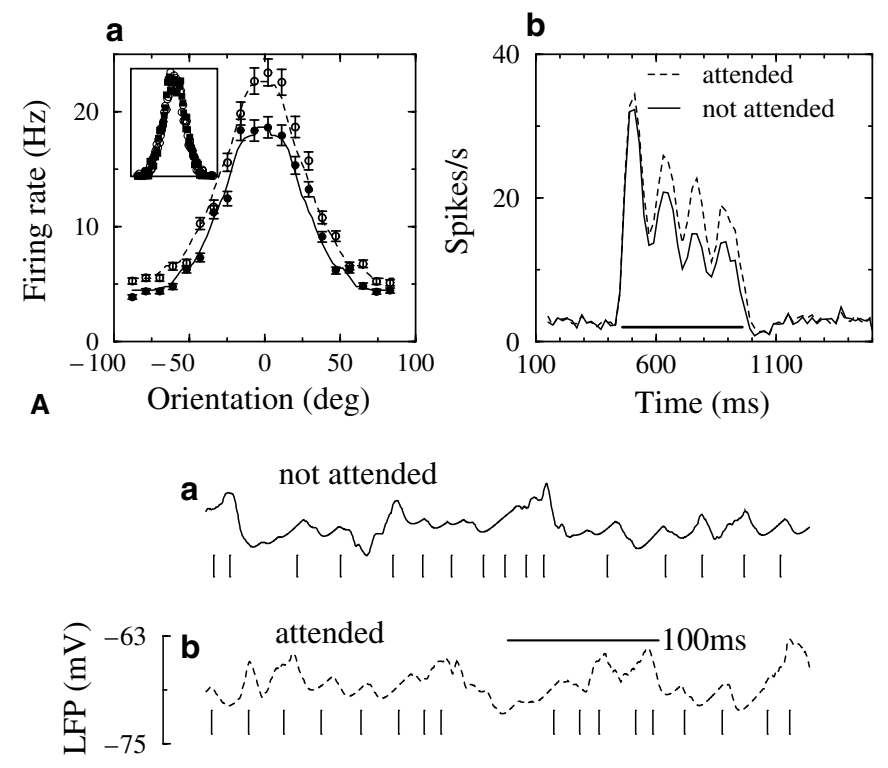

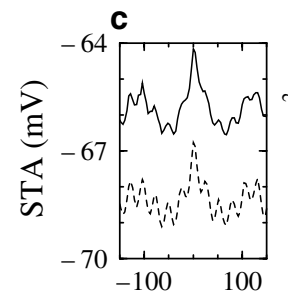

B time difference (ms)
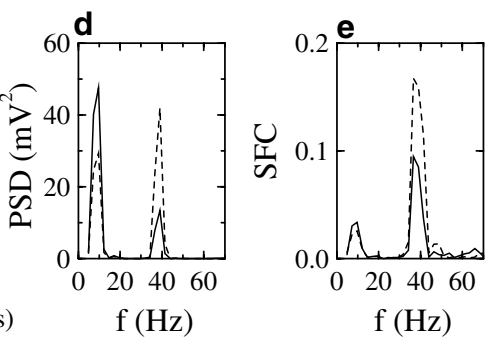

Fig. 10. Modulation of inhibitory synchrony in model simulations reproduced attentional modulation of V4 neurons observed in experiment. (A) Model of attentional modulation in McAdams and Maunsell [49]. The neuron received synchronous inhibitory input. (a) Firing rate as a function of stimulus orientation for two conditions: (solid lines, filled symbols) attention was directed away from the receptive field, $\sigma_{\mathrm{IV}}=8 \mathrm{~ms}$ and (dashed lines, open symbols) attention was directed into the receptive field, $\sigma_{\mathrm{IV}}=7 \mathrm{~ms}$. Inset: The two curves coalesced when the asymptotic firing rate was subtracted and the residual of the solid line was rescaled by a factor 1.2 along the $y$-axis. (b) Temporal dynamics of attentional modulation of the firing rate. The bar indicates the presence of a driving current representing the presence of a stimulus in the receptive field. (Solid line) Attention directed away from receptive field, $\sigma_{\mathrm{IV}}=3 \mathrm{~ms}$ and (dashed line) into the receptive field, $\sigma_{\mathrm{IV}}=2 \mathrm{~ms}$. (B) Model of attentional modulation reported in Fries et al. [29]. A neuron received synchronous inhibitory input in the gamma-frequency range, and excitatory input in the theta-frequency range. For the solid lines attention was directed away from the receptive field, $\sigma_{\mathrm{IV}}=5 \mathrm{~ms}$, and for the dashed lines attention was directed into the receptive field, $\sigma_{\mathrm{IV}}=4 \mathrm{~ms}$. ( $\mathrm{a}-\mathrm{b}$, top) The local field potential (LFP) and (bottom) a spike train from one neuron. (c) The spike-triggered average (STA) of the LFP, the solid line was shifted by $+2 \mathrm{mV}$ for clarity. (d) Power spectrum density (PSD) of the STA. (e) The spike field coherence (SFC). The full set of parameter values is given in Appendix A.

curves, and the resulting curve in the non-attended state was rescaled by a factor 1.2. After these manipulations, the curves for the attended and non-attended states overlapped (Fig. 10Aa, inset), indicating, as observed experimentally, that the mean and width of the Gaussian had not changed. Similar results (data not shown) were obtained by decreasing the spike-time dispersion from $\sigma_{\mathrm{IV}}=3$ to $2 \mathrm{~ms}$. The firing rate in the non-preferred 
orientation increased from $1.3 \mathrm{~Hz}$ to $4.2 \mathrm{~Hz}$ in the attended state, whereas the firing rate in the preferred orientation increased from $13.6 \mathrm{~Hz}$ to $24.7 \mathrm{~Hz}$.

\subsubsection{Computer Experiment II: Fig. 8 of McAdams and Maunsell [49]}

Next, the temporal dynamics of attentional modulation in this figure were obtained using $\sigma_{\mathrm{IV}}=2 \mathrm{~ms}$ for the attended state and $\sigma_{\mathrm{IV}}=3 \mathrm{~ms}$ for the non-attended state (Fig. 10Ab). The background firing rate was $3 \mathrm{~Hz}$ and was not modulated by attention. There was an $8 \mathrm{~Hz}$ temporal modulation, with a peak firing rate at stimulus onset equal to $34.4 \mathrm{~Hz}$. The firing-rate ratio between attended and non-attended state increased from 1 at stimulus onset to approximately 1.5 later in the trial, compatible with the experimental data.

\subsubsection{Computer Experiment III: Fig. 1 of Fries et al. [29]}

In the experiments of Fries et al. [29], the spikes from a neuron on one electrode were used to calculate the spiketriggered average (STA) of the LFP on another electrode. In the model, the LFP was estimated from the subthreshold membrane potential of a neuron that received theta-frequency excitatory and a gamma-frequency inhibitory synaptic inputs (Fig. 10B). The STA in the model was calculated based on the spike train from another neuron receiving the same synaptic inputs. The coherence of the inhibition was varied between the attended and nonattended state $\left(\sigma_{\mathrm{IV}}=5 \mathrm{~ms}\right.$ to $4 \mathrm{~ms} ; a_{\mathrm{IV}}=5$ to 6$)$. There were no changes to the excitatory synaptic drive received by the neuron. However, the mean level of depolarization was varied to keep the mean firing rate constant (as explained in Appendix A, this also helps in estimating the SFC). We also analyzed two frequency bands, $5<f<$ $15 \mathrm{~Hz}$ (theta) and $34<f<44 \mathrm{~Hz}$ (gamma). The SFC in the theta range was $21 \%$ less in the attended compared to the non-attended state, whereas the SFC in the gammafrequency range more than doubled $(114 \%$ increase, Fig. 10Be). Our model does not predict the exact dynamics of the LFP. The mean, phase and amplitude of this model LFP was different from the extracellularly recorded signals. Specifically, the gamma oscillations visible during the attended condition in Fig. 10Ba were much less pronounced than in experiment (Fig. 1B of Ref. [29]). The modeled STA looks different from measured STA for the same reason. However, the SFC is a ratio between the power spectrum of the STA and that of the LFP and might therefore be less sensitive to the differences between the modeled and experimental LFP. Indeed, the changes in the model SFC with attention are qualitatively similar to those reported in Ref. [29]. There were quantitative differences: the increase in SFC with attention was of the order of $10 \%$ in experiment, whereas in the model it was an order of magnitude larger. These differences could possibly be resolved by a more detailed model that incorporates the electrical behavior of the extracellular medium in order to estimate the LFP more accurately.

\section{Discussion}

\subsection{Summary}

The firing rate and coherence of a neuron can be modulated by the degree of synchrony of the inhibitory input. In the model investigated here, the mean number of inputs on each cycle, $a_{\mathrm{IV}}$, was a key parameter in determining how the neuron's response properties depended on synchrony. The $a_{\mathrm{IV}}$ is proportional to the number of neurons in the presynaptic network that are activated and the synaptic reliability (the probability that a presynaptic action potential results in a postsynaptic potential). The network activation is controlled by neuromodulators, such as acetylcholine, or by activation of metabotropic glutamate receptors [78]. It depends also on the extent of the chemical and gap-junction couplings within local networks [1] and, possibly, on longer range connections between different local networks $[83,84]$. So $a_{\mathrm{IV}}$ may reflect a combination of physiological parameters.

\subsubsection{Input synchrony modulates firing rate}

The firing rate increased when input synchrony was increased by reducing $\sigma_{\mathrm{IV}}$. When $\sigma_{\mathrm{IV}}$ was modulated dynamically, the change in firing rate was immediate and robust across trials. This implies that temporal dynamics of firing rate modulation is determined by how fast a presynaptic network can be activated, and how fast network activation results in an increase in synchrony. Interneuron networks can modulate their synchrony in a few oscillation cycles - on the order of $100 \mathrm{~ms}$ for gamma-frequency range activity [79] - and the firing rate response is able to follow these rapid synchrony modulations. Rapid modulation of the firing rate observed in the cortex can therefore be due to the temporal dynamics of the stimulus as well as synchrony. In addition to serving as a way to amplify the significance of information represented in a neural population, inhibitory synchrony provides an alternative pathway for cortical information transmission that can operate in parallel with changes in activity. Our investigation also revealed that other statistical properties of the input, besides $\sigma_{\mathrm{IV}}$, such as the oscillation frequency and $\mathrm{CV}_{T}$, could also dynamically modulate the output firing rate (data not shown).

\subsubsection{Input synchrony modulates the sensitivity and gain of $f-I$ curves}

The $f-I$ curves were characterized in terms of sensitivity, the weakest inputs to which the neuron will respond, and the gain, the rate of change of the firing rate with input amplitude. By increasing input synchrony, the neuron responded to weaker stimuli, and its gain increased so that the same increase in stimulus strength would result in a stronger increase of the firing rate. The relative size of the shift in sensitivity compared with the change in gain depended on $a_{\mathrm{IV}}$. For small $a_{\mathrm{IV}} \sim 10$, the change in gain dominated, whereas for larger $a_{\mathrm{IV}} \sim 50$, the shift in sensi- 
tivity dominated. In the latter case, saturation was observed: when the firing rate was approximately equal to the oscillation frequency, it did not increase further when either the input was made stronger or when the input synchrony was increased. It should be noted that the unitary strength of the inhibitory inputs was normalized such that the mean (time-averaged) inhibitory conductance remained constant when $a_{\mathrm{IV}}$ was varied. This allowed us to distinguish the effects of an increase in mean inhibitory conductance with $a_{\mathrm{IV}}$ from the effects of the reduced variability in the input with $a_{\mathrm{IV}}$.

\subsubsection{Input synchrony modulates spike coherence with the network oscillation}

We measured the vector strength (VS) of the output spike train with respect to the oscillatory activity of the inhibitory input. Changes in the VS reflected the behavior of the SFC in the gamma-frequency range, but VS was easier to calculate and more robust than the SFC. The VS increased with input synchrony and it even did so when the firing rate remained the same.

\subsubsection{Input synchrony modulates the variability of spike trains within and across trials}

We determined the Fano factor (FF), representing the variability of the spike count across trials and the coefficient of variation $(\mathrm{CV})$ - the variability of interspike intervals during a trial. Generally, FF and $\mathrm{CV}$ decreased with increasing input synchrony. We found non-monotonic behavior of $\mathrm{FF}$ and $\mathrm{CV}$ as a function of the firing rate for $a_{\mathrm{IV}} \sim 50$. Furthermore, the FF was sensitive to the jitter across trials in the phase of the oscillation at the start of the trial (or stimulus onset), whereas the $\mathrm{CV}$ was not sensitive to this phase.

\subsubsection{Firing rate modulation with input synchrony was most prominent for gamma-frequency oscillation}

The increase in firing rate induced by changing $\sigma_{\mathrm{IV}}$ was maximal for $f_{\text {osc }}=40 \mathrm{~Hz}$ because of the time-constant of the inhibitory synapses. Networks of interneurons synchronize in the same frequency range $[28,81]$, indicating that the synchrony of their activity is well suited to modulate the firing rate of the pyramidal cells in cortex.

\subsection{Can attention modulate the synchrony of interneuron networks?}

Although the synchronization dynamics of inhibitory networks has been studied extensively using model simulations $[4,5,73,75,77,86-88]$, the focus has almost exclusively been on the stationary state, rather than dynamic changes in synchrony. We found two types of networks whose synchrony can be changed by neuromodulators or excitatory neurotransmitters [78]. These results will be presented elsewhere; we summarize them here briefly. First, in a purely inhibitory network, synchrony can be modulated by increasing excitation to a part of the network. The acti- vated neurons increase their firing rate and synchronize and reduce the activity of the other group of interneurons [79]. Hence, the mean activity of the network that projects to a postsynaptic neuron, like the one studied here, remains approximately constant. Synchrony can be modulated using this mechanism on time scales as short as $100 \mathrm{~ms}$. Second, in a mixed excitatory and inhibitory network, synchrony can be modulated by activating the interneuron network when the inhibitory and excitatory neurons are mode-locked to each other. In that case $[8,9,73]$, synchronized excitatory activity recruits inhibitory activity that temporarily shuts down the excitatory activity. When the inhibition decays the excitatory neurons become active again and the cycle starts over. Activation of interneuron networks by neuromodulators may increase their synchrony, in turn increasing excitatory synchrony, but without altering the mean firing rate of individual neurons [78].

\subsection{Gain modulation $f-I$ curves}

The statistics of the synaptic inputs determine the sensitivity and gain of the $f-I$ curve. Three mechanisms have been proposed for how multiplicative gain changes can be achieved [10,14,24,26,38,52,54,58,65,76,85]. We briefly summarize them and discuss how they relate to gain modulation by inhibitory synchrony.

The response properties of neurons are different when they are driven by a supra- or infra-threshold currents [76]. In the former case neurons are tonically active and fluctuations in the input will not alter the firing rate, whereas in the latter case action potentials are induced by fluctuations (referred to as a fluctuation-dominated state [76]). The firing rates for neurons in the fluctuation-dominated state can be increased by either reducing the distance of the mean membrane potential to threshold, or by increasing the amplitude of the voltage fluctuations. For each of the mechanisms discussed below the neuron operates in the fluctuation regime, but the way that fluctuations increase or decrease the distance to threshold is different.

\subsubsection{Gain modulation by balanced synaptic inputs}

Under in vivo conditions neurons receive a constant barrage of excitatory and inhibitory inputs [67]. The synaptic inputs are called balanced when the effective reversal potential of the sum of excitatory and inhibitory inputs is equal to the neuron's resting membrane potential (leak reversal potential). By proportionally scaling the rates of excitatory and inhibitory inputs the amplitude of the voltage fluctuations can be modulated while maintaining a constant mean membrane potential. In the balanced mode the neuron is driven by fluctuations: the larger the fluctuations, the higher the firing rate. Chance et al. [14] found multiplicative gain modulation of the $f-I$ curves of neurons recorded in vitro experiments. Interestingly, an increase in balanced activity decreased the gain $[10,11,76]$. The reason for this somewhat counterintuitive result is that the 
increase in input conductance dominates the increase in conductance variance, resulting in an amplitude reduction of voltage fluctuations. The saturation of dendritic nonlinearities can further enhance the change in gain obtained with balanced inputs [58]. In a modeling study in which the excitatory and inhibitory fluctuations were independently varied, varying the amplitude of the inhibitory conductances was more effective than varying the amplitude of the excitatory conductances [26].

\subsubsection{Gain modulation by tonic inhibition and excitation}

Tonic inhibition by itself did not lead to multiplicative gain modulation $[24,38]$. However, when tonic inhibition was applied in combination with either excitatory or inhibitory Poisson spike train inputs, changes in gain as well as shifts in sensitivity were observed [52,85]. Recently Murphy and Miller [54] showed that changes in tonic excitation and inhibition can lead to approximate multiplicative gain modulation of cortical responses when the nonlinearity of the thalamic contrast response is taken into account.

\subsubsection{Gain modulation by correlations}

When a neuron is in a fluctuation-dominated state and receives inputs from different neurons, it is sensitive to correlations between these neurons. Stronger correlations lead to an increase in the amplitude of voltage fluctuations, hence to an increase in firing rate $[65,66]$, because the mean input conductance is not altered by correlations, as was the case for balanced synaptic inputs.

\subsubsection{Gain modulation by inhibitory synchrony}

Changing input synchrony for small $a_{\mathrm{IV}}$ values resulted in a gain change of the $f-I$ curve. This mechanism is complementary to gain modulation by correlation. There are two different aspects of synchrony, the degree of coincidence - how many neurons fire at approximately the same time, $a_{\mathrm{IV}}$, and the precision - the temporal dispersion of the neurons that fire together, $\sigma_{\mathrm{IV}}$. The number of neurons $a_{\mathrm{IV}}$ roughly corresponds to the degree of correlation. Changing $\sigma_{\text {IV }}$ resulted both in a change of the distance to threshold as well as the amplitude of fluctuations. However, the 'threshold' here is a conductance threshold: The neuron spiked when the inhibitory conductance became smaller than a threshold value. The mean distance of the minimum inhibitory conductance from threshold and the fluctuations about this value determined the firing rate change. We are not aware of any mechanisms that have been proposed to selectively change the level of balanced input or degree of correlations in the network. In contrast, mechanisms have been proposed to modulate the synchrony of the network, the parameter $\sigma_{\mathrm{IV}}[79]$.

\subsection{Relation between attention and modulation of inhibitory synchrony}

More than a decade ago Crick and Koch proposed a link between oscillatory synchrony and attentional process- ing [19]. This led to a model in which excitatory neurons representing stimuli in the focus of attention produced correlated spike trains [56]. In their model, interneurons in cortical area V4 were activated by correlated spike trains and in turn suppressed V4 neurons responsive to stimuli outside the focus of attention; hence, the synchronized interneurons suppressed rather than enhanced activity. In the mechanism explored here, interneurons are active irrespective of the attentional state, and their degree of synchrony modulates the responsiveness of V4 output neurons. The time-course of attentional modulation of neural responses was also studied by Deco and coworkers $[18,22]$ but the synchrony of interneuron networks was not taken into account.

Under the assumption that attention acts by increasing the synchrony of interneuron networks, our model predicts that: (1) attention increases the coherence of spike trains with the local field potential; (2) the firing rate can increase with attention or remain the same depending on the stimulus strength; (3) attention can lead to a multiplicative gain change of firing rate response curves or to a shift in the sensitivity, depending on the extent of interneuron network activation by attention and the stimulus. Thus, changes in interneuron synchrony could potentially underlie a variety of seemingly unrelated observations. The size of the firing rate modulation predicted by this model agrees quantitatively with experimental observations (Sections 3.5 and Appendix A). However, these results do not provide direct evidence that modulation of inhibitory synchrony is, in fact, responsible for the observed attentional effects. In the following we discuss specific predictions that derive from this hypothesis.

McAdams and Maunsell [49] observed multiplicative gain modulation of the orientation tuning curves of V4 neurons. We could reproduce these results quantitatively by changing $\sigma_{\mathrm{IV}}$. The values of $\sigma_{\mathrm{IV}}$ corresponding to the attended and non-attended state were not unique and we could obtain the same results with different combinations. The only constraint was that $\sigma_{\mathrm{IV}}$ in the attended state had to be lower than in the non-attended state. Multiplicative gain modulation of $f-I$ curves could simply be the consequence of a power law between $f$ and $I[37,51]$ and account for the contrast independence of orientation selectivity [2]. We could not fit our $f-I$ curves with a power-law and changing $\sigma_{\mathrm{IV}}$ both changed the gain and shifted the sensitivity - the gain change was not purely multiplicative. We could account for the results by McAdams and Maunsell because the firing rate in response to a non-preferred stimulus - the so-called asymptote-was modulated by attention even though the background activity-the firing rate without stimulus-was not affected. Only the modulation of the firing rate tuning curve above this asymptote was multiplicative. Hence, both a shift in sensitivity and a change in gain were required. The change in gain in the model only needed to be multiplicative over a limited range of the input current $I$. 
Recent experiments were performed to determine whether attention would modulate the gain of the firing rate response, or whether the response properties could be interpreted as a shift in sensitivity $[48,63,64]$. The firing rate elicited in response to a visual stimulus was measured as a function of stimulus contrast. The firing rate versus contrast had a sigmoidal shape and saturated. Gain modulation would imply that the saturation rate was increased by attention. This was not observed in the experiments and the changes were more consistent with a shift in sensitivity. Hence, depending on the specifics of the experimental protocol, attention can either increase sensitivity $[48,63,64]$ or gain $[49,82]$. Our model predicts that this behavior is correlated with the connectivity and degree of activation of the interneuron network.

McAdams and Maunsell also reported that the attentional modulation of the firing rate reached its stationary value $500 \mathrm{~ms}$ after the onset of the response to the stimulus (Appendix A). We could reproduce this by assuming that the $\sigma_{\text {IV }}$ value changed gradually from its background value (here equal to the $\sigma_{\mathrm{IV}}$ in the non-attended state) to its value in the attended state. There was no change in $\sigma_{\mathrm{IV}}$ if there was no stimulus. The model used here does not make predictions about the temporal dynamics of $\sigma_{\mathrm{IV}}$ since that is a network property. However, if the change in firing rate is the result of modulating the inhibitory synchrony it implies that the stimulus needs to activate the interneuron network either directly in a bottom up fashion or indirectly through top down inputs.

Fries et al. [29] reported that attention induced an increase in the gamma-frequency range coherence of the neuron's spike train with the LFP which was accompanied by only small changes in its firing rate. We observed that a decrease in $\sigma_{\mathrm{IV}}$ results in an increased coherence. This is expected based on general arguments. The amplitude of the inhibitory conductance waveform, defined as the distance between maximum and minimum value, increased with input synchrony (decreasing $\sigma_{\mathrm{IV}}$ ). The spike timing precision of a neuron, which is directly related to the VS and $\sigma_{\phi}$, increased with the amplitude of the periodic driving current [72]. The same holds for periodic and aperiodic stimulus waveforms in vivo $[7,60]$. The small change in firing rate is indicative of saturation. We observed saturation for large $a_{\mathrm{IV}} \sim 50$ when the neuron's firing rate was close to the oscillation frequency of the inhibitory drive. The saturation of the firing rate was associated with a reduction in response variability on a given trial $(\mathrm{CV})$ and across trials (FF).

There are other potential explanations for saturation. It can be due to the activation of intrinsic currents or increases in input conductance associated with synaptic background activity. These saturation effects would, however, in general not cause the reduction of response variability predicted by our model. In those experiments where attention did modulate the firing rate response, no concomitant reduction of response variability was observed in recordings of V4 neurons [50].

\subsection{Open problems and future work}

The attentional modulation of the firing rate of V4 neurons has been studied with two stimuli [44,62], a preferred stimulus that elicited a vigorous and robust response and a non-preferred stimulus that elicited a weaker response. When both stimuli were presented simultaneously in the neuron's receptive field, the firing rate was intermediate between the responses to each of the stimuli presented separately, rather than the sum of the two responses as a linear model would predict. This result was explained by a simple model proposed by Reynolds et al. [62]. Each stimulus activated a presynaptic population of neurons (in V2 in this case) that projected excitation and inhibition to the V4 neuron. The inhibitory component of the projection for the non-preferred stimulus was stronger and reduced the response to the preferred stimulus when both stimuli were presented simultaneously, consistent with the experimental observation. Their hypothesis was that attention would enhance synaptic efficacy of the presynaptic population of neurons representing the stimulus in the focus of attention, without increasing the activity of the presynaptic population. In their model, attention shifts the response toward the one that would be expected when the attended stimulus was presented alone, as indeed is observed experimentally.

In our model, the attentional enhancement in synaptic efficacy corresponds to an increase in synchrony of the inhibitory projection. This correctly predicts an increase in firing rate when attention is directed toward the preferred stimulus compared with attention directed outside the receptive field. However, when attention was directed toward the non-preferred stimulus, our model would still predict an increase in firing rate, albeit smaller, rather than the decrease observed in experiment. The underlying assumption was that attention operates bottom up from V2 to V4. An alternative hypothesis is that attentional modulation of synchrony is top down and operates on interneuron networks in V4 itself. This would predict that input synchrony increases when preferred stimuli in the receptive field are attended, but that synchrony decreases when the non-preferred stimuli are attended.

Neurons in vivo receive massive amounts of excitatory and inhibitory inputs [57]. Here we assumed that part of the inhibitory input was temporally modulated and observed that the firing rate could saturate when at the inhibitory oscillation frequency. The saturation rates observed during experiments may not correspond to those predicted by the model. It is likely that part of the excitation is also temporally modulated, allowing for the possibility that the neuron could saturate at rates determined by the time scale of temporal patterning of excitatory inputs. These inputs do not need to be oscillatory. Furthermore, in our model neuron there were no adaptation currents and there also was no synaptic coupling between neurons. An important issue for future study is 
how temporally modulated excitatory and inhibitory inputs derived from a network interact with intrinsic time-scales of the neuron (such as adaptation currents) to determine the saturation rate.

\section{Appendix A}

\section{A.1. Review of in vivo experimental results}

The roman numerals correspond to the subsections in Section 3.5.

Experiment I: McAdams and Maunsell [49] recorded the response of a V4 neuron to Gabor patches (a sinusoidal grating multiplied by a 2-dimensional Gaussian density) that were presented for $500 \mathrm{~ms}$ in its receptive field. The spatial frequency, color and size of the Gabor patch were chosen to elicit maximal responses. The contrast of the patch varied sinusoidally with a frequency of $4 \mathrm{~Hz}$. During the experiment the orientation of the Gabor patch was varied systematically. The responses of the neuron were recorded when the animal had to focus attention into the receptive field ("attended") and when it had to focus onto a different Gabor patch at equal eccentricity away from the receptive field ("non-attended").

The mean firing rate of about $75 \%$ of the cells that responded to this stimulus, could be fitted by a Gaussianshaped orientation tuning curve in both the attended and non-attended states. There were four fitting parameters, the mean, width and amplitude of the Gaussian density and the asymptote (the firing rate in response to the least preferred stimulus orientation). We focused on Fig. 4 in [49], which showed population-averaged orientation-tuning curves. The asymptote was approximately $5 \mathrm{~Hz}$ in the nonattended state and increased slightly in the attended state. The amplitude of the Gaussian increased by $22 \%$, going to $15 \mathrm{~Hz}$ in the attended state from $12 \mathrm{~Hz}$ in the nonattended state. The mean and width of the Gaussian did not change significantly with attention.

Experiment II: Fig. 8 of [49] shows the temporal dynamics of attentional modulation averaged across all responsive neurons. The background firing rate, before and after stimulus presentation, was $3.6 \mathrm{~Hz}$. It did not vary significantly with attention. There was an $8 \mathrm{~Hz}$ stimuluslocked modulation in the firing rate with a peak firing rate of $35 \mathrm{~Hz}$ at stimulus onset. The ratio of stimulus-induced firing in the attended state to that in the unattended state increased from unity at stimulus onset to about 1.5, $500 \mathrm{~ms}$ after stimulus onset. The time course of this ratio was similar across different stimulus orientations.

Experiment III: A similar attentional paradigm was used in the experiments by Fries et al. [29]. They presented a pure luminance sinusoidal grating at $100 \%$ contrast and optimal orientation in the receptive field of a V4 neuron for a random interval that lasted between $500 \mathrm{~ms}$ and $5000 \mathrm{~ms}$. They recorded neural activity using 4 electrodes that were spaced 650 or $900 \mu \mathrm{m}$ apart. Multi-unit activity (elicited by the stimulus in the receptive field) was recorded on one electrode and the local field potential was recorded on a different electrode. The spike-triggered average (STA) on the LFP was calculated during stimulus presentation when the animal focused attention into the receptive field and when attention was directed away from the receptive field. The first $300 \mathrm{~ms}$ after stimulus onset were discarded prior to their analysis. Changes of coherence with attentional state were assessed using the power spectrum density (PSD) of the STA, and the spike field coherence (SFC), which is the PSD of the STA normalized by the PSD of the LFP. The spectrum was divided into two frequency bands: $f<10 \mathrm{~Hz}$ (low frequency or theta) and $35<f<60$ $\mathrm{Hz}$ (high frequency or gamma). The SFC in the low frequency range decreased by $23 \%$ going from the nonattended to attended state, whereas the SFC in the gamma frequency range increased by $19 \%$. The mean spike rate in the multi-unit recording did not change by more than $15 \%$ with attentional state.

\section{A.2. Neuron model}

The equation for the membrane potential of the neuron was

$C_{m} \frac{\mathrm{d} V}{\mathrm{~d} t}=-I_{N a}-I_{K}-I_{L}-I_{\mathrm{syn}}+I+C_{m} \xi$,

with the leak current $I_{L}=g_{L}\left(V-E_{L}\right)$, the sodium current $I_{\mathrm{Na}}=g_{\mathrm{Na}} m_{\infty}^{3} h\left(V-E_{\mathrm{Na}}\right)$, the potassium current: $I_{K}=g_{K} n^{4}\left(V-E_{K}\right)$, and the synaptic current $I_{\text {syn }}$ as described in Section 2. The intrinsic noise $\xi$ had zero mean and variance $2 D$, and $I$ was the injected current. The channel kinetics were given in terms of $m, n$, and $h$. They satisfied the following first-order kinetic equations,

$\frac{\mathrm{d} x}{\mathrm{~d} t}=\zeta\left(\alpha_{x}(1-x)-\beta_{x} x\right)$.

Here $x$ labels the different kinetic variables $m, n$, and $h$, and $\zeta=5$ was a dimensionless time-scale that was used to tune the temperature-dependent speed with which the channels opened or closed. The rate constants were [86],

$$
\begin{aligned}
& \alpha_{m}=\frac{-0.1(V+35)}{\exp (-0.1(V+35))-1}, \\
& \beta_{m}=4 \exp (-(V+60) / 18), \\
& \alpha_{h}=0.07 \exp (-(V+58) / 20), \\
& \beta_{h}=\frac{1}{\exp (-0.1(V+28))+1}, \\
& \alpha_{n}=\frac{-0.01(V+34)}{\exp (-0.1(V+34))-1}, \\
& \beta_{n}=0.125 \exp (-(V+44) / 80) .
\end{aligned}
$$

We made the approximation that $m$ took the asymptotic value $m_{\infty}(V(t))=\alpha_{m} /\left(\alpha_{m}+\beta_{m}\right)$ instantaneously. The standard set of values for the conductances used in this paper was $g_{N a}=35, g_{K}=9$, and $g_{L}=0.1$ (in $\mathrm{mS} / \mathrm{cm}^{2}$ ), and we took $E_{N a}=55 \mathrm{mV}, E_{K}=-90 \mathrm{mV}$, and $E_{L}=-65 \mathrm{mV}$. 
The membrane capacitance was $C_{m}=1 \mu \mathrm{F} / \mathrm{cm}^{2} . \Delta g_{\text {inh }}$ has units $\mathrm{mS} / \mathrm{cm}^{2} ; I$ is in $\mu \mathrm{A} / \mathrm{cm}^{2} ; D$ is $\mathrm{mV}^{2} / \mathrm{ms} ; f_{\text {osc }}$ is in $\mathrm{Hz} ; P$ and $\sigma_{\mathrm{IV}}$ are in ms; and $a_{\mathrm{IV}}$ is dimensionless.

\section{A.3. Relation between the spike field coherence and the vector strength}

The spike-triggered average (STA) of the LFP is the average LFP waveform around an output spike. The $\mathrm{SFC}$ is the ratio of the power spectrum density (PSD) of the STA and that of the LFP (see Methods). To obtain some intuition of the meaning of the SFC consider a simple example. Let the LFP be a $40 \mathrm{~Hz}$ cosine, the PSD then has a single peak at $40 \mathrm{~Hz}$. Suppose neuron 1 produces action potentials on some cycles of the LFP at a fixed phase $\phi$. The STA is a $40 \mathrm{~Hz}$ cosine but shifted over $\phi$, yielding a PSD with a peak at $40 \mathrm{~Hz}$ (the PSD is not sensitive to the phase of the cosine). The SFC at $40 \mathrm{~Hz}$ is one and zero elsewhere. Suppose that the spike train of neuron 2 forms a homogeneous Poisson process with the same mean firing rate as neuron 1. The spikes are uncorrelated with the LFP and uniformly distributed in time. The STA would be equal to the mean of the LFP, zero in this case, and the PSD at $40 \mathrm{~Hz}$ would be zero, yielding an SFC equal to zero.

When there is jitter in the phase, with standard deviation $\sigma_{\phi}$, the SFC is $\exp \left(-4 \pi^{2} \sigma_{\phi}^{2}\right)$. For this situation the SFC is completely determined by the phase jitter. The arrival times of the volleys of inhibitory inputs are known in the model simulations, hence the phase of spike times with respect to the oscillation are known. The phase is a cyclical variable, which means that $\sigma_{\phi}$ is not a good measure of the phase jitter (it depends on the mean phase and the jitter), but the vector strength is, $\mathrm{VS}=|\langle\exp (\mathrm{i} 2 \pi \phi)\rangle|=$ $\sqrt{\langle\cos (2 \pi \phi)\rangle^{2}+\langle\sin (2 \pi \phi)\rangle^{2}}$. For our example, we obtain $\mathrm{VS}=\exp \left(-4 \pi^{2} \sigma_{\phi}^{2}\right)=\mathrm{SFC}$, such that $\mathrm{VS}=1$ for perfect phase-locking (neuron 1) and VS is zero for a uniform phase distribution such as would be the case for neuron 2. Our analysis yields a potentially important insight into the experimental results of Fries et al. [29]. When the amplitude of the LFP is increased, but the spike times stay exactly the same, the amplitude of the STA increases proportionally and the SFC stays the same. However, when the phase jitter of the spike times decreases at the same time, the SFC will increase. Previously we found that for noisy neurons driven by sinusoidal current the jitter decreases when the amplitude of the current increases [80]. This follows because the spike-time jitter is proportional to $\mathrm{d} V / \mathrm{d} t$ at the action potential threshold [13], and because $\mathrm{d} V / \mathrm{d} t$ is proportional to the stimulus amplitude. The LFP is in general not a cosine but contains other frequency components, hence, the VS is not equal to the SFC under general conditions, but changes in the VS faithfully represent changes in the coherence in the gamma-frequency range which is the subject of this study.
These theoretical results for the STA are based on averages over the full distribution of spike times. In computational experiments the STA is sampled using a finite number of spike times. For $\sigma_{\phi}=0$, the STA obtained using only one spike is equal to its theoretical value. However, for neuron 2, the STA for one spike would be a cosine, and the SFC would be one. The theoretical STA, equal to zero, can only be obtained by adding many cosines with random phases. This explains the counterintuitive results for the SFC calculated based on Fig. 2: The firing rate was so low, that the STA was not correctly sampled for the given trial length. The calculation of the SFC is illustrated in Fig. 11.
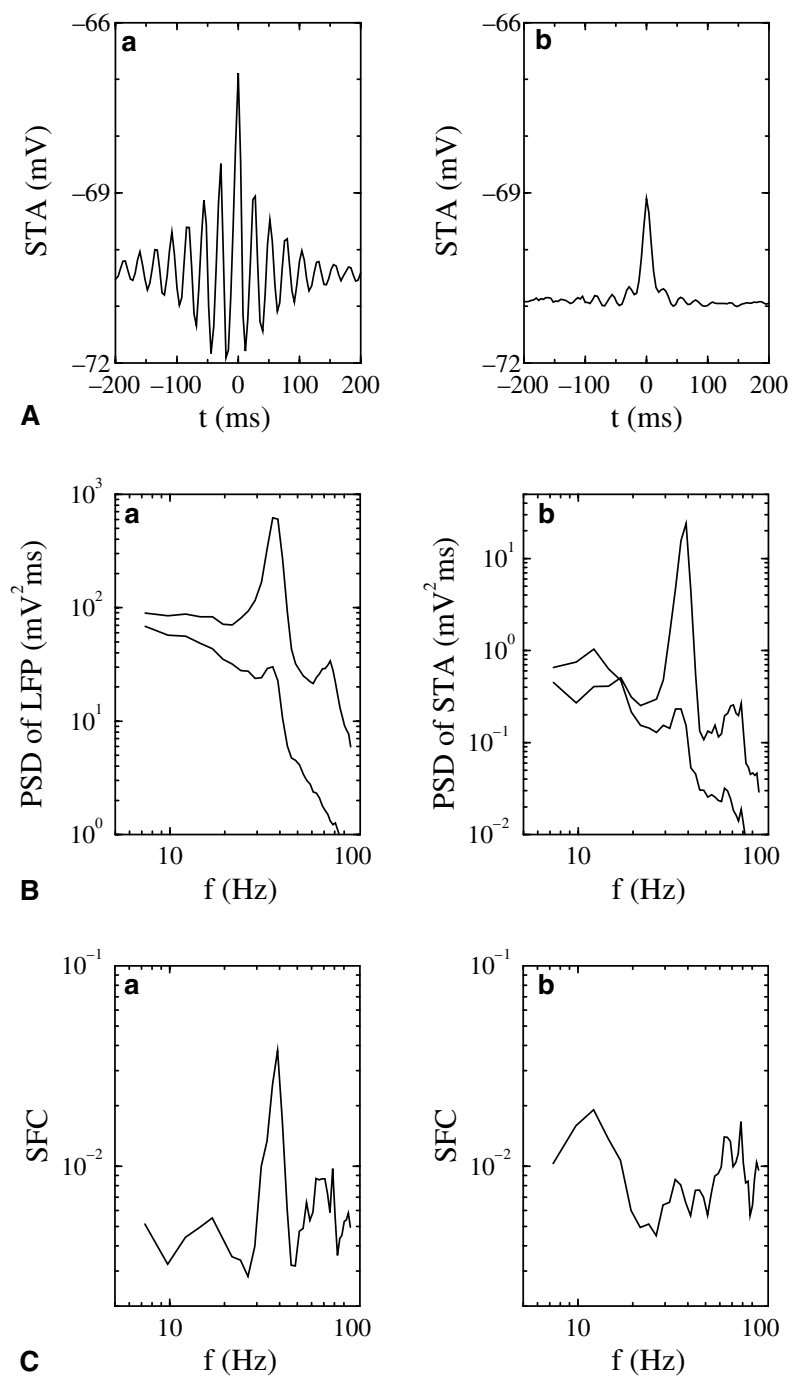

Fig. 11. Calculation of the Spike Field Coherence (SFC) for the model parameters used for Fig. 2. The model parameters are the same except that for $\sigma_{\mathrm{IV}}=8 \mathrm{~ms}$ we took $I=6.0 \mu \mathrm{A} / \mathrm{cm}^{2}$. (A) Spike triggered average (STA) of the LFP for (a) $\sigma_{\mathrm{IV}}=2 \mathrm{~ms}$ and (b) $8 \mathrm{~ms}$. (B) The power spectrum (PSD) of (a) the LFP and (b) the STA for (top) $\sigma_{\mathrm{IV}}=2 \mathrm{~ms}$ and (bottom) $8 \mathrm{~ms}$. (C) SFC for (a) $\sigma_{\mathrm{IV}}=2 \mathrm{~ms}$ and (b) $8 \mathrm{~ms}$. 


\section{A.4. Parameter values for the figures}

Fig. 2: The neuron was driven by a synchronous inhibitory input with $a_{\mathrm{IV}}=25, \Delta g_{\text {inh }}=0.044 \mathrm{mS} / \mathrm{cm}^{2}, \tau_{\text {inh }}=$ $10 \mathrm{~ms}, P=26.10 \mathrm{~ms}, f_{\text {osc }}=38.3 \mathrm{~Hz}, \mathrm{CV}_{T}=0.095, I=4.0$ $\mu \mathrm{A} / \mathrm{cm}^{2}, D=0.08 \mathrm{mV}^{2} / \mathrm{ms}$. For $t<300$ and $t>700$, the spike time dispersion $\sigma_{\mathrm{IV}}$ was $8 \mathrm{~ms}$, and, for $300 \leqslant$ $t \leqslant 700$, it was $\sigma_{\mathrm{IV}}=2 \mathrm{~ms}$. The subthreshold membrane potential in (b) was obtained by reducing the injected current to $I=1.0 \mu \mathrm{A} / \mathrm{cm}^{2}$ to prevent action potentials. The firing rate histogram was calculated based on 500 trials.

Fig. 3: The neuron was driven by a synchronous inhibitory input with $a_{\mathrm{IV}}=10, \Delta g_{\text {inh }}=0.11 \mathrm{mS} / \mathrm{cm}^{2}, \tau_{\text {inh }}=$ $10 \mathrm{~ms}, P=26.10 \mathrm{~ms}, f_{\text {osc }}=38.3 \mathrm{~Hz}, \mathrm{CV}_{T}=0.095, I=2.4$ $\mu \mathrm{A} / \mathrm{cm}^{2}, \quad D=0.04 \mathrm{mV}^{2} / \mathrm{ms}$. For $t<1000$ and $t>2000$, the spike time dispersion $\sigma_{\mathrm{IV}}$ was $4 \mathrm{~ms}$, and, for $1000 \leqslant$ $t \leqslant 2000$, it was $\sigma_{\mathrm{IV}}=2 \mathrm{~ms}$. There was also a temporally homogeneous excitatory input with rate $\lambda_{\mathrm{exc}}=1000 \mathrm{~Hz}$, $\Delta g_{\text {exc }}=0.02 \mathrm{mS} / \mathrm{cm}^{2}$ and $\tau_{\text {exc }}=2 \mathrm{~ms}$. The subthreshold membrane potential in (b) was obtained by reducing current to $I=-2.0 \mu \mathrm{A} / \mathrm{cm}^{2}$ to prevent action potentials. The firing rate histogram was calculated based on 500 trials.

Fig. 4: The neuron was driven by a synchronous inhibitory input with $a_{\mathrm{IV}}=50, \Delta g_{\text {inh }}=0.022 \mathrm{mS} / \mathrm{cm}^{2}, \tau_{\text {inh }}=$ $10 \mathrm{~ms}, P=26.10 \mathrm{~ms}, f_{\text {osc }}=38.3 \mathrm{~Hz}, \mathrm{CV}_{T}=0.0, D=0.0$ $\mathrm{mV}^{2} / \mathrm{ms}$. Parameters were on a two-dimensional grid defined by: $I=2.0$ to $I=7.5 \mu \mathrm{A} / \mathrm{cm}^{2}$ in steps of $0.1 \mu \mathrm{A} /$ $\mathrm{cm}^{2}$ and $\sigma_{\mathrm{IV}}=1 \mathrm{~ms}$ to $\sigma_{\mathrm{IV}}=6 \mathrm{~ms}$ in steps of $0.5 \mathrm{~ms}$. For each parameter value, statistics were over 20 trials of $3000 \mathrm{~ms}$ length.

Fig. 5: The parameters of the inhibitory and excitatory drive were the same for Fig. 3, except that $\sigma_{\mathrm{IV}}$ and $I$ were varied on a two-dimensional grid defined by: $I=0.0$ to $I=5.0 \mu \mathrm{A} / \mathrm{cm}^{2}$ in steps of $0.1 \mu \mathrm{A} / \mathrm{cm}^{2}$ and $\sigma_{\mathrm{IV}}=1 \mathrm{~ms}$ to $\sigma_{\mathrm{IV}}=10 \mathrm{~ms}$ in steps of $1 \mathrm{~ms}$. For each parameter value, statistics were over 20 trials of $3000 \mathrm{~ms}$ length.

Fig. 6(A): Parameter values were $P=26.08 \mathrm{~ms}$, $f_{\text {osc }}=38.35 \mathrm{~Hz}, \mathrm{CV}_{T}=0, a_{\mathrm{IV}}=10, g_{\text {inh }}=0.11 \mathrm{mS} / \mathrm{cm}^{2}$, $\tau_{\text {inh }}=10 \mathrm{~ms}, D=0 \mathrm{mV}^{2} / \mathrm{ms}$. From left to right, $\sigma_{\mathrm{IV}}=1,2$, 3,4 , and $5 \mathrm{~ms}$. Inset, data for $\sigma_{\mathrm{IV}}=2,3,4$, and $5 \mathrm{~ms}$ was rescaled according to $f \rightarrow f / \lambda_{f}$ and $I \rightarrow\left(I-\Delta_{I}\right)$ to coalesce with the curve for $\sigma_{\mathrm{IV}}=1 \mathrm{~ms}$. (B) Parameter values were $P=26.08 \mathrm{~ms}, f_{\text {osc }}=38.35 \mathrm{~Hz}, \mathrm{CV}_{T}=0, a_{\mathrm{IV}}=50, g_{\text {inh }}=$ $0.022 \mathrm{mS} / \mathrm{cm}^{2}, \tau_{\text {inh }}=10 \mathrm{~ms}, D=0 \mathrm{mV}^{2} / \mathrm{ms}$. From left to right, $\sigma_{\mathrm{IV}}=1,3$ and $5 \mathrm{~ms}$. A sigmoidal function, $f(I)=\frac{A}{2}\left(1+\tanh \left(\lambda_{I}\left(I-\Delta_{I}\right)\right)\right)$, was fitted to the $f-I$ curves. In (A-B) a transient of $100 \mathrm{~ms}$ was discarded before analysis. The firing rate was then calculated over a $50 \mathrm{~s}$ time interval.

Fig. 7: Parameter values were $P=26.08 \mathrm{~ms}, f_{\text {osc }}=$ $38.35 \mathrm{~Hz}, \mathrm{CV}_{T}=0, \tau_{\mathrm{inh}}=10 \mathrm{~ms}, \sigma_{\mathrm{IV}}=1 \mathrm{~ms}$, and $D=$ $0 \mathrm{mV}^{2} / \mathrm{ms}$. In (A, C) $a_{\mathrm{IV}}=10, g_{\text {inh }}=0.11 \mathrm{mS} / \mathrm{cm}^{2}$ and (B, D) $a_{\mathrm{IV}}=50, g_{\text {inh }}=0.022 \mathrm{mS} / \mathrm{cm}^{2}$. From left to right (A, B) or as indicated by the direction of the arrow
(C, D), the input rate was $100,300,500,700$ and 900 EPSPs per second. A transient of $50 \mathrm{~ms}$ was discarded before analysis. The firing rate was then calculated over 20 trials of $3 \mathrm{~s}$ duration.

Fig. 9: Parameter values were $P=26.08 \mathrm{~ms}, f_{\text {osc }}=$ $38.35 \mathrm{~Hz}, C V_{T}=0, a_{\mathrm{IV}}=25, g_{\text {inh }}=0.044 \mathrm{mS} / \mathrm{cm}^{2}, \tau_{\text {inh }}=$ $10 \mathrm{~ms}, I=5.0 \mu \mathrm{A} / \mathrm{cm}^{2}, D=0 \mathrm{mV}^{2} / \mathrm{ms}$. Mean firing rate was calculated over $50 \mathrm{~s}$ after discarding a $100 \mathrm{~ms}$ transient. The dynamical range was defined as the ratio of the firing rate for $\sigma_{\mathrm{IV}}=4 \mathrm{~ms}$ divided by that for $\sigma_{\mathrm{IV}}=10 \mathrm{~ms}$ provided the latter is non-zero.

Fig. 10(Aa): Neurons received orientation-tuned input current, $I=I_{O}+A_{O} \exp \left(-\psi^{2} / 2 \sigma_{\psi}^{2}\right)$ with $I_{O}=5.5 \mu \mathrm{A} / \mathrm{cm}^{2}$, $A_{O}=1.3 \mu \mathrm{A} / \mathrm{cm}^{2}, \sigma_{\psi}=30^{\circ}$. $\psi$ took values between $-90^{\circ}$ and $+90^{\circ}$ with discretization step $\Delta \psi=9 / 4^{\circ}$. The firing rate was obtained from pre-calculated $f-I$ curves using interpolation. Parameters were: $\sigma_{\mathrm{IV}}=8 \mathrm{~ms}$ for attention directed away and $\sigma_{\mathrm{IV}}=7 \mathrm{~ms}$ for attention directed into the receptive field; $P=26.08 \mathrm{~ms}, f_{\text {osc }}=38.35 \mathrm{~Hz}, \mathrm{CV}_{T}=0, a_{\mathrm{inh}}=25$, $\Delta g_{\text {inh }}=0.044 \mathrm{mS} / \mathrm{cm}^{2}, \tau_{\text {inh }}=10 \mathrm{~ms}, D=0 \mathrm{mV}^{2} / \mathrm{ms}$. To mimic neuronal variability, normally distributed noise with a standard deviation equal to $5 \%$ of the firing rate was added to the firing rate. Solid curves are a 4-point running average, only every fourth point is shown in the graph.

$(\mathrm{Ab})$ Neurons received a time-dependent driving current $I(t)$ and an inhibitory synchronous drive with time-varying parameters $a_{\mathrm{IV}}(t)$. In the non-attended state $\sigma_{\mathrm{IV}}=3 \mathrm{~ms}$ and it was $\sigma_{\mathrm{IV}}=2 \mathrm{~ms}$ in the attended state. For $t<500$, the current was $I(t)=0$; for $500 \leqslant t<1000$, it was $I(t)=$ $0.8+0.56[\sin (2 \pi t / 250)]^{2}+2.4 \min [(t-500) / 350,1] ;$ for $1000 \leqslant t<1100$, it was $I(t)=3.2[1-(t-1000) / 100]$; and for $t \geqslant 1100$, the current was zero. For $t<500$, the inhibitory activity was $a_{\mathrm{IV}}=5$; for $500 \leqslant t<850, a_{\mathrm{IV}}=5+$ $20[(t-500) / 350]$; for $850 \leqslant t<1000$, it was $a_{\mathrm{IV}}=25$; for $1000 \leqslant t<1100$, it was $a_{\mathrm{IV}}=25-20[(t-1000) / 1000]$; and for $t \geqslant 1100$, it was $a_{\mathrm{IV}}=5 . I$ was in $\mu \mathrm{A} / \mathrm{cm}^{2}, t$ and $\sigma_{\mathrm{IV}}$ in $\mathrm{ms}$. Other parameters were $P=26.39 \mathrm{~ms}, f_{\mathrm{osc}}=$ $37.90 \mathrm{~Hz}, C V_{T}=0.11, D=0.4 \mathrm{mV}^{2} / \mathrm{ms}$.

(B) The neuron received synchronous inhibitory and excitatory drives. Parameters for the excitatory drive were $P=116 \mathrm{~ms}, f_{\text {osc }}=8.62 \mathrm{~Hz}, \quad \mathrm{CV}_{T}=0, a_{E V}=25, \sigma_{E V}=$ $30 \mathrm{~ms}$. For the inhibitory drive they were $P=26.08 \mathrm{~ms}$, $f_{\text {osc }}=38.35 \mathrm{~Hz}, \mathrm{CV}_{T}=0$, with, for the non-attended state, (Ba) $\sigma_{\mathrm{IV}}=5 \mathrm{~ms}, a_{\mathrm{IV}}=5, I=1.6 \mu \mathrm{A} / \mathrm{cm}^{2}$; and for the attended state, $(\mathrm{Bb}) \sigma_{\mathrm{IV}}=4 \mathrm{~ms}, a_{\mathrm{IV}}=6, I=1.8 \mu \mathrm{A} / \mathrm{cm}^{2}$. In both cases, $D=0 \mathrm{mV}^{2} / \mathrm{ms}$. The local field potential was calculated by hyperpolarizing the neuron (the injected current was reduced to $I=0.0 \mu \mathrm{A} / \mathrm{cm}^{2}$ ). (Bc) Spike triggered average was calculated based on a 10 second long LFP waveform of which the first $200 \mathrm{~ms}$ was discarded as a transient. Sampling rate was $5 \mathrm{kHz}$ (temporal resolution was $0.2 \mathrm{~ms}$ ). (Bd) Power spectrum density was calculated based on the STA sampled at 4096 points at a temporal resolution of $0.2 \mathrm{~ms}$, there were $n_{\mathrm{fft}}=2048$ points in the Fourier transform. 


\section{References}

[1] Y. Amitai, J. Gibson, M. Beierlein, S. Patrick, B. Connors, D. Golomb, The spatial dimensions of electrically coupled networks of interneurons in the neocortex, J. Neurosci. 22 (2002) 4142-4152.

[2] J. Anderson, I. Lampl, D. Gillespie, D. Ferster, The contribution of noise to contrast invariance of orientation tuning in cat visual cortex, Science 290 (2000) 1968-1972.

[3] I. Aradi, V. Santhakumar, K. Chen, I. Soltesz, Postsynaptic effects of GABAergic synaptic diversity: regulation of neuronal excitability by changes in IPSC variance, Neuropharmacology 43 (2002) 511-522.

[4] I. Aradi, I. Soltesz, Modulation of network behaviour by changes in variance in interneuronal properties, J. Physiol. 538 (2002) 227-251.

[5] M. Bartos, I. Vida, M. Frotscher, A. Meyer, H. Monyer, J. Geiger, P. Jonas, Fast synaptic inhibition promotes synchronized gamma oscillations in hippocampal interneuron networks, Proc. Natl. Acad. Sci. 99 (2002) 13222-13227.

[6] M. Beierlein, J. Gibson, B. Connors, A network of electrically coupled interneurons drives synchronized inhibition in neocortex, Nat. Neurosci. 3 (2000) 904-910.

[7] M. Berry, D. Warland, M. Meister, The structure and precision of retinal spike trains, Proc. Natl. Acad. Sci. 94 (1997) 5411-5416.

[8] C. Borgers, N. Kopell, Synchronization in networks of excitatory and inhibitory neurons with sparse random connectivity, Neural Comput. 15 (2003) 509-538.

[9] N. Brunel, X. Wang, What determines the frequency of fast network oscillations with irregular neural discharges? I. Synaptic dynamics and excitation-inhibition balance, J. Neurophysiol. 90 (2003) 415-430.

[10] A. Burkitt, Balanced neurons: analysis of leaky integrate-and-fire neurons with reversal potentials, Biol. Cybern. 85 (2001) 247-255.

[11] A. Burkitt, H. Meffin, D. Grayden, Study of neuronal gain in a conductance-based leaky integrate-and-fire neuron model with balanced excitatory and inhibitory synaptic input, Biol. Cybern. 89 (2003) 119-125.

[12] P. Bush, T. Sejnowski, Inhibition synchronizes sparsely connected cortical neurons within and between columns in realistic network models, J. Comput. Neurosci. 3 (1996) 91-110.

[13] G. Cecchi, M. Sigman, J. Alonso, L. Martinez, D. Chialvo, M. Magnasco, Noise in neurons is message dependent, Proc. Natl. Acad. Sci. 97 (2000) 5557-5561.

[14] F. Chance, L. Abbott, A. Reyes, Gain Modulation from Background Synaptic Input, Neuron 35 (2002) 773-782.

[15] S. Cobb, E. Buhl, K. Halasy, O. Paulsen, P. Somogyi, Synchronization of neuronal activity in hippocampus by individual GABAergic interneurons, Nature 378 (1995) 75-78.

[16] C. Connor, J. Gallant, D. Preddie, D.C. Van Essen, Responses in area V4 depend on the spatial relationship between stimulus and attention, J. Neurophysiol. 75 (1996) 1306-1308.

[17] C. Connor, D. Preddie, J. Gallant, D.C. Van Essen, Spatial attention effects in macaque area V4, J. Neurosci. 17 (1997) 3201-3214.

[18] S. Corchs, G. Deco, Large-scale neural model for visual attention: integration of experimental single-cell and fMRI data, Cerebral Cortex 12 (2002) 339-348.

[19] F. Crick, C. Koch, Some reflections on visual awareness, Cold Spring Harb. Symp. Quant. Biol. 55 (1990) 953-962.

[20] P. Dayan, L. Abbott, Theoretical Neuroscience, MIT press, Cambridge, Massachusetts, 2001.

[21] M. Deans, J. Gibson, C. Sellitto, B. Connors, D. Paul, Synchronous activity of inhibitory networks in neocortex requires electrical synapses containing connexin36, Neuron 31 (2001) 477-485.

[22] G. Deco, O. Pollatos, J. Zihl, The time course of selective visual attention: theory and experiments, Vision Res. 42 (2002) 2925-2945.

[23] M. Diesmann, M. Gewaltig, A. Aertsen, Stable propagation of synchronous spiking in cortical neural networks, Nature 402 (1999) 529-533.

[24] B. Doiron, A. Longtin, N. Berman, L. Maler, Subtractive and divisive inhibition: effect of voltage-dependent inhibitory conductances and noise, Neural Comput. 13 (2001) 227-248.
[25] J. Fell, G. Fernandez, P. Klaver, C. Elger, P. Fries, Is synchronized neuronal gamma activity relevant for selective attention? Brain Res. Rev. 42 (2003) 265-272.

[26] J.-M. Fellous, M. Rudolph, A. Destexhe, T. Sejnowski, Variance detection and gain modulation in an in vitro model of in vivo activity, Neuroscience 122 (2003) 811-829.

[27] D. Ferster, K. Miller, Neural mechanisms of orientation selectivity in the visual cortex, Annu. Rev. Neurosci. 23 (2000) 441-471.

[28] A. Fisahn, F. Pike, E. Buhl, O. Paulsen, Cholinergic induction of network oscillations at $40 \mathrm{~Hz}$ in the hippocampus in vitro, Nature 394 (1998) 186-189.

[29] P. Fries, J. Reynolds, A. Rorie, R. Desimone, Modulation of oscillatory neuronal synchronization by selective visual attention, Science 291 (2001) 1560-1563.

[30] J. Frost, Comparison of intracellular potentials and ECoG activity in isolated cerebral cortex, Electroencephalogr. Clin. Neurophysiol. 23 (1967) 89-90.

[31] M. Galarreta, S. Hestrin, A network of fast-spiking cells in the neocortex connected by electrical synapses, Nature 402 (1999) 72-75.

[32] M. Galarreta, S. Hestrin, Electrical synapses between GABA-releasing interneurons, Nat. Rev. Neurosci. 2 (2001) 425-433.

[33] M. Galarreta, S. Hestrin, Spike transmission and synchrony detection in networks of GABAergic interneurons, Science 292 (2001) 22952299.

[34] M. Gazzaniga, R. Ivry, G. Mangun, Cognitive Neuroscience, Norton, New York, 1998.

[35] J. Gibson, M. Beierlein, B. Connors, Two networks of electrically coupled inhibitory neurons in neocortex, Nature 402 (1999) 75-79.

[36] H. Greenside, E. Helfand, Numerical integration of stochastic differential equations, Bell Syst. Technol. J. 60 (1981) 1927.

[37] D. Hansel, C. van Vreeswijk, How noise contributes to contrast invariance of orientation tuning in cat visual cortex, J. Neurosci. 22 (2002) 5118-5128.

[38] G. Holt, C. Koch, Shunting inhibition does not have a divisive effect on firing rates, Neural Comput. 9 (1997) 1001-1013.

[39] S. Hormuzdi, I. Pais, F. LeBeau, S. Towers, A. Rozov, E. Buhl, M. Whittington, H. Monyer, Impaired electrical signaling disrupts gamma frequency oscillations in connexin 36-deficient mice, Neuron 31 (2001) 487-495.

[40] J. José, P. Tiesinga, J. Fellous, E. Salinas, T. Sejnowski, Is attentional gain modulation optimal at gamma frequencies?, Soc Neurosci. Abstr. 28 (2002).

[41] J. José, P. Tiesinga, J.-M. Fellous, E. Salinas, T. Sejnowski, Synchronization as a mechanism for attentional modulation, Soc. Neurosci. Abstr. 27 (2001).

[42] C. Koch, Biophysics of Computation, Oxford university press, New York, 1999.

[43] T. Koos, J. Tepper, Inhibitory control of neostriatal projection neurons by GABAergic interneurons, Nat Neurosci. 2 (1999) 467472.

[44] S. Luck, L. Chelazzi, S. Hillyard, R. Desimone, Neural mechanisms of spatial selective attention in areas V1, V2, and V4 of macaque visual cortex., J. Neurophysiol. 77 (1997) 24-42.

[45] W. Lytton, T. Sejnowski, Simulations of cortical pyramidal neurons synchronized by inhibitory interneurons, J. Neurophysiol. 66 (1991) 1059-1079.

[46] Z. Mainen, T. Sejnowski, Reliability of spike timing in neocortical neurons, Science 268 (1995) 1503-1506.

[47] K. Mardia, P. Jupp, Directional Statistics, Wiley, New York, 2000.

[48] J.C. Martinez Trujillo, S. Treue, Attentional modulation strength in cortical area MT depends on stimulus contrast, Neuron 35 (2002) 365-370.

[49] C. McAdams, J. Maunsell, Effects of attention on orientation-tuning functions of single neurons in macaque cortical area V4, J. Neurosci. 19 (1999) 431-441.

[50] C. McAdams, J. Maunsell, Effects of attention on the reliability of individual neurons in monkey visual cortex, Neuron 23 (1999) 765773 . 
[51] K. Miller, T. Troyer, Neural noise can explain expansive, power-law nonlinearities in neural response functions, J. Neurophysiol. 87 (2002) 653-659.

[52] S. Mitchell, R. Silver, Shunting inhibition modulates neuronal gain during synaptic excitation, Neuron 38 (2003) 433-445.

[53] T. Moore, K. Armstrong, Selective gating of visual signals by microstimulation of frontal cortex, Nature 421 (2003) 370-373.

[54] B. Murphy, K. Miller, Multiplicative gain changes are induced by excitation or inhibition alone, J. Neurosci. 23 (2003) 10040-10051.

[55] A. Neiman, X. Pei, D. Russell, W. Wojtenek, L. Wilkens, F. Moss, Synchronization of the noisy electro-sensitive cells in the paddlefish, Phys. Rev. Lett. 82 (1999) 660-663.

[56] E. Niebur, C. Koch, C. Rosin, An oscillation-based model for the neuronal basis of attention, Vision Res. 33 (1993) 2789-2802.

[57] D. Pare, A. Destexhe, Impact of network activity on the integrative properties of neocortical pyramidal neurons in vivo, J. Neurophysiol. 81 (1999) 1531-1547.

[58] S. Prescott, Y. De Koninck, Gain control of firing rate by shunting inhibition: Roles of synaptic noise and dendritic saturation, Proc. Natl. Acad. Sci. 100 (2003) 2071-2081.

[59] W. Press, S. Teukolsky, W.T. Vetterling, B.P. Flannery, Numerical Recipes, Cambridge University Press, Cambridge, 1992.

[60] D. Reich, J. Victor, B. Knight, T. Ozaki, E. Kaplan, Response variability and timing precision of neuronal spike trains in vivo, J. Neurophysiol. 77 (1997) 2836-2841.

[61] R. Rensink, The dynamics representation of scenes, Visual Cognit. 7 (2000) $17-42$.

[62] J. Reynolds, L. Chelazzi, R. Desimone, Competitive mechanisms subserve attention in macaque areas V2 and V4, J. Neurosci. 19 (1999) 1736-1753.

[63] J. Reynolds, R. Desimone, Interacting roles of attention and visual salience V4, Neuron 37 (2003) 853-863.

[64] J. Reynolds, T. Pasternak, R. Desimone, Attention increases sensitivity of V4 neurons, Neuron 26 (2000) 703-714.

[65] E. Salinas, T. Sejnowski, Impact of correlated synaptic input on output variability in simple neuronal models, J. Neurosci. 20 (2000) 6193-6209.

[66] E. Salinas, T. Sejnowski, Integrate-and-fire neurons driven by correlated stochastic input, Neural Comput. 14 (2002) 2111-2155.

[67] M. Shadlen, W. Newsome, The variable discharge of cortical neurons: implications for connectivity, computation, and information coding, J. Neurosci. 18 (1998) 3870-3896.

[68] R. Shapley, M. Hawken, D. Ringach, Dynamics of orientation selectivity in the primary visual cortex and the importance of cortical inhibition, Neuron 38 (2003) 689-699.

[69] D.J. Simons, Current approaches to change blindness, Visual Cognit. 7 (2000) 1-15.

[70] P. Steinmetz, A. Roy, P. Fitzgerald, S. Hsiao, K. Johnson, E. Niebur, Attention modulates synchronized neuronal firing in primate somatosensory cortex, Nature 404 (2000) 187-190.
[71] G. Tamas, E. Buhl, A. Lorincz, P. Somogyi, Proximally targeted GABAergic synapses and gap junctions synchronize cortical interneurons, Nat. Neurosci. 3 (2000) 366-371.

[72] P. Tiesinga, Precision and reliability of periodically and quasiperiodically driven integrate-and-fire neurons, Phys. Rev. E 65 (2002) 041913.

[73] P. Tiesinga, J.-M. Fellous, J. José, T. Sejnowski, Computational model of carbachol-induced delta, theta and gamma oscillations in the hippocampus, Hippocampus 11 (2001) 251-274.

[74] P. Tiesinga, J.-M. Fellous, J. José, T. Sejnowski, Information transfer in entrained neocortical neurons, Network 13 (2002) 41-66.

[75] P. Tiesinga, J. José, Robust gamma oscillations in networks of inhibitory hippocampal interneurons, Network 11 (2000) 1-23.

[76] P. Tiesinga, J. José, T. Sejnowski, Comparison of current-driven and conductance-driven neocortical model neurons with Hodgkin-Huxley voltage-gated channels, Phys. Rev. E 62 (2000) 8413-8419.

[77] P. Tiesinga, W.-J. Rappel, J.V. José, Synchronization in networks of noisy interneurons, in: J. Bower (Ed.), Computational Neuroscience, Plenum Press, New York, 1998, pp. 555-559.

[78] P. Tiesinga, T. Sejnowski, Attentional modulation of synchrony in cortical networks, Soc. Neurosci. Abstr. 28 (2002) 558.10.

[79] P. Tiesinga, T. Sejnowski, Rapid temporal modulation of synchrony by competition in cortical interneuron networks, Neural Comput. 16 (2004) 251-275.

[80] P. Tiesinga, P. Thomas, J. Fellous, T. Sejnowski, Reliability, Precision and the Neural Code, Society for Neuroscience Abstract 27 (2001).

[81] R. Traub, M. Whittington, S. Colling, G. Buzsaki, J. Jeffreys, Analysis of gamma rhythms in the rat hippocampus in vitro and in vivo, J. Physiol. 493 (1996) 471-484.

[82] S. Treue, J.C. Martinez Trujillo, Feature-based attention influences motion processing gain in macaque visual cortex, Nature 399 (1999) $575-579$.

[83] T. Tucker, L. Katz, Recruitment of local inhibitory networks by horizontal connections in layer $2 / 3$ of ferret visual cortex, J. Neurophysiol. 89 (2003) 501-512.

[84] T. Tucker, L. Katz, Spatiotemporal patterns of excitation and inhibition evoked by the horizontal network in layer $2 / 3$ of ferret visual cortex, J. Neurophysiol. 89 (2003) 488-500.

[85] D. Ulrich, Differential arithmetic of shunting inhibition for voltage and spike rate in neocortical pyramidal cells, Eur. J. Neurosci. 18 (2003) 2159-2165.

[86] X. Wang, G. Buzsáki, Gamma oscillation by synaptic inhibition in a hippocampal interneuronal network model, J. Neurosci. 16 (1996) 6402-6413.

[87] J. White, C. Chow, J. Ritt, C. Soto-Treviño, N. Kopell, Synchronization and oscillatory dynamics in heterogeneous, mutually inhibited neurons, J. Comput. Neurosci. 5 (1998) 5-16.

[88] M. Whittington, R. Traub, J. Jeffreys, Synchronized oscillations in interneuron networks driven by metabotropic glutamate receptor activation, Nature 373 (1995) 612-615. 